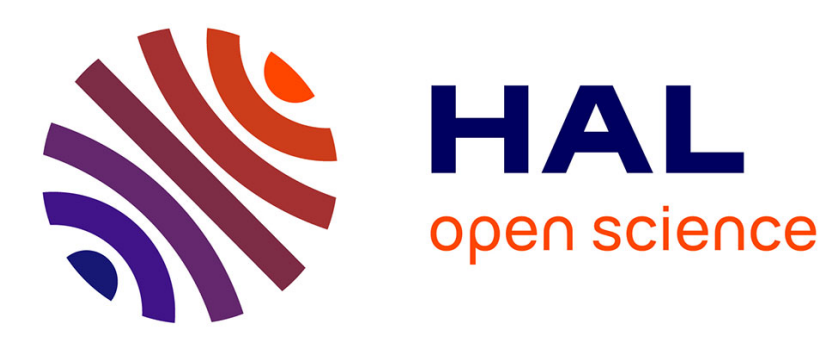

\title{
New boundary conditions for the computation of the apparent stiffness of statistical volume elements
}

\author{
Sébastien Brisard, Karam Sab, Luc Dormieux
}

\section{To cite this version:}

Sébastien Brisard, Karam Sab, Luc Dormieux. New boundary conditions for the computation of the apparent stiffness of statistical volume elements. Journal of the Mechanics and Physics of Solids, 2013, 61 (12), pp.2638-2658. 10.1016/j.jmps.2013.08.009 . hal-00871767

\section{HAL Id: hal-00871767 \\ https://hal-enpc.archives-ouvertes.fr/hal-00871767}

Submitted on 10 Oct 2013

HAL is a multi-disciplinary open access archive for the deposit and dissemination of scientific research documents, whether they are published or not. The documents may come from teaching and research institutions in France or abroad, or from public or private research centers.
L'archive ouverte pluridisciplinaire HAL, est destinée au dépôt et à la diffusion de documents scientifiques de niveau recherche, publiés ou non, émanant des établissements d'enseignement et de recherche français ou étrangers, des laboratoires publics ou privés. 


\title{
New boundary conditions for the computation of the apparent stiffness of Statistical Volume Elements
}

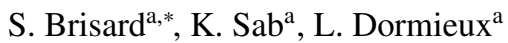 \\ ${ }^{a}$ Université Paris-Est, Laboratoire Navier (UMR 8205), CNRS, ENPC, IFSTTAR, F-77455 Marne-la-Vallée
}

\begin{abstract}
We present a new auxiliary problem for the determination of the apparent stiffness of a Statistical Volume Element (SVE). The SVE is embedded in an infinite, homogeneous reference medium, subjected to a uniform strain at infinity, while tractions are applied to the boundary of the SVE to ensure that the imposed strain at infinity coincides with the average strain over the SVE. The main asset of this new auxiliary problem resides in the fact that the associated Lippmann-Schwinger equation involves without approximation the Green operator for strains of the infinite body, which is translation-invariant and has very simple, closed-form expressions. Besides, an energy principle of the Hashin and Shtrikman type can be derived from this modified Lippmann-Schwinger equation, allowing for the computation of rigorous bounds on the apparent stiffness. The new auxiliary problem requires a cautious mathematical analysis, because it is formulated in an unbounded domain. Observing that the displacement is irrelevant for homogenization purposes, we show that selecting the strain as main unknown greatly eases this analysis. Finally, it is shown that the apparent stiffness defined through these new boundary conditions "interpolates" between the apparent stiffnesses defined through static and kinematic uniform boundary conditions, which casts a new light on these two types of boundary conditions.
\end{abstract}

Keywords: Apparent stiffness, Boundary conditions, Effective stiffness, Homogenization, Lippmann-Schwinger equation

\section{Introduction}

The determination of the macroscopic properties of heterogeneous materials can be carried out by means of micromechanical models such as the model of Mori and Tanaka (1973) (see also Benveniste, 1987; Ponte Castañeda and Willis, 1995), the model of Maxwell (McCartney and Kelly, 2008; McCartney, 2010), the self-consistent model (Walpole, 1969; Kröner, 1977) or the generalized self-consistent model (Christensen and Lo, 1979; Hervé and Zaoui, 1993). These are invaluable tools, which provide semi-analytical (or even closed-form) estimates; besides, material non-linearities can be accomodated (Suquet, 1997). However, it is well-known that they fail to account for the finest details of the microstructure. This is due to the fact that most of them are based on the elementary solution to the problem of one single inhomogeneity, embedded in an infinite, homogeneous matrix (Eshelby, 1957). Since the inhomogeneity under consideration in this auxiliary problem is isolated, microstructural correlations can only be approximately incorporated. In cases where a more faithful representation of the microstructure is needed, it is therefore essential to resort to numerical homogenization, which provides accurate estimates derived from full-field computations.

Within the framework of numerical homogenization, the effective stiffness of heterogeneous materials is usually estimated as the limit of the apparent stiffness of Statistical Volume Elements [SVEs, using the terminology introduced by Ostoja-Starzewski (2006)] of growing size (Sab, 1992). In turn, the apparent stiffness is derived from the solution to an auxiliary boundary value problem which states the elastic equilibrium of the SVE. The present paper is devoted to

\footnotetext{
${ }^{*}$ Corresponding author.

Email addresses: sebastien.brisard@ifsttar.fr (S. Brisard), karam.sab@enpc.fr (K. Sab), luc.dormieux@enpc.fr (L. Dormieux)
} 
the issue of selecting appropriate boundary conditions for this auxiliary problem. Three types of boundary conditions are frequently adopted, namely static and kinematic uniform boundary conditions (Hill, 1963, 1967; Mandel, 1972), and periodic boundary conditions (Gusev, 1997, among others).

In the case of linear elasticity and kinematic uniform boundary conditions, the Lippmann-Schwinger equation is an alternative (equivalent) formulation of the auxiliary problem (Zeller and Dederichs, 1973). Upon introduction of a so-called reference medium, the classical boundary value problem of elasticity with the displacement as main unknown is replaced with a unique integral equation with the polarization as main unknown.

In comparison with the initial boundary value problem, the equivalent integral equation has a number of assets, both in periodic and random homogenization. In periodic homogenization for example, the structure of the equation lends itself to efficient numerical treatments in the Fourier space (Moulinec and Suquet, 1994, 1998; Brisard and Dormieux, 2010, 2012). The resulting schemes can be extended to non-linear problems (Michel et al., 2001). In the present paper, periodic boundary conditions are not adopted, and discretization of the Lippmann-Schwinger equation can no longer benefit from formulations in the Fourier space. However, discretization of this equation in the real space can still be carried out in an efficient way under certain circumstances. This is true of e.g. random matrix-inclusions composites. Indeed, the polarization in the matrix vanishes if the latter is selected as reference medium. Then, the polarization needs only be approximated in the inclusions (by e.g. piecewise polynomials), which results in a significant reduction of the number of degrees of freedom. This is the basis of the Equivalent Inclusion Method (Moschovidis and Mura, 1975), a variational version of which will be proposed by the authors in a future publication.

Solving numerically the auxiliary problem with kinematic uniform boundary conditions would require the discretization of a Lippmann-Schwinger equation involving the Green operator for strains of a bounded domain. Such an approach has two shortcomings. First, this operator is known for very specific shapes of the bounded domain only. Second, it is not translation-invariant; as a consequence, the influence pseudo-tensors, which characterize the interaction between two inclusions, would depend on the position of both inclusions. This would result in a costly assembly of the linear system resulting from the discretization of the Lippmann-Schwinger equation.

To overcome these shortcomings, it is necessary to substitute the Green operator of the infinite domain (whole space) to the Green operator of finite domains. This substitution effectively amounts to embedding the SVE in an infinite medium, with imposed strain at infinity (see Fig. 3). The Equivalent Inclusion Method is in fact formulated in this spirit. Since the Green operator of the infinite domain is translation-invariant, the influence pseudo-tensors of two inclusions depend on their relative position only, thus easing assembly of the underlying linear system.

However, the resulting Lippmann-Schwinger equation is not well-suited to numerical homogenization, as the corresponding boundary conditions do not allow for the specification of neither the macroscopic strain nor the macroscopic stress. Although it is still possible to define the apparent stiffness associated with these boundary conditions [see Fond et al. (2002) and Sec. 3.2 in the present paper], the resulting estimates cannot be regarded as bounds, as the principle of Hashin and Shtrikman is lost.

In the present paper, we introduce a new auxiliary problem, with mixed boundary conditions, and the associated modified Lippmann-Schwinger equation, which involves the Green operator of the infinite domain. As previously suggested by Willis (1977), this operator is applied to the fluctuations of the polarization. However, Willis regarded the resulting integral equation as an approximation of the Lippmann-Schwinger equation associated to kinematic uniform boundary conditions for infinitely large SVEs. By contrast, in this paper, we regard this equation as the exact Lippmann-Schwinger equation associated to the new, mixed boundary conditions, combining imposed strain at infinity and imposed tractions at the boundary of the finite-size SVE (see Fig. 2).

This new auxiliary problem with mixed boundary conditions has a number of assets. First, the loading parameter is the macroscopic strain (unlike the problem depicted in Fig. 3). This results in a direct definition of the corresponding apparent stiffness; it differs from the apparent stiffness based on static or kinematic uniform boundary conditions. Second, minimum potential and complementary energy principles can be derived, which in turn allow for the mathematical analysis of the well-posedness of the new auxiliary problem, as well as the elementary properties of the apparent stiffness. Third, an energy principle of the Hashin and Shtrikman (1962a) type can be derived; under classical restrictions on the stiffness of the reference medium, it is therefore possible to exhibit bounds on the apparent stiffness. As the underlying Green operator is translation-invariant, this energy principle lends itself to direct discretization in a numerical setting. Finally, the new definition of the apparent stiffness "interpolates" between the two classical definitions based on static and kinematic uniform boundary conditions. Indeed, when the reference (embedding) medium becomes infinitely soft (resp. stiff), the apparent stiffness associated with static (resp. kinematic) uniform 
boundary conditions is recovered. For finite stiffness reference media, the apparent stiffness associated with mixed boundary conditions is bounded by these two limit apparent stiffnesses.

The remainder of this paper is organized as follows. In Sec. 2, the definitions of static and kinematic uniform boundary conditions are first recalled. Then, the new, mixed boundary conditions are introduced. The mathematical properties of the resulting auxiliary problem, and the associated apparent stiffness are stated without proof. The essential property is the asymptotic consistency: like standard problems with static and kinematic uniform boundary conditions, the apparent stiffness tends to the effective stiffness as the size of the SVE grows to infinity. This justifies the relevance of the new boundary conditions.

In Sec. 3, we present the rationale behind these new boundary conditions. The starting point is the LippmannSchwinger equation, which is first recalled, together with the corresponding Hashin and Shtrikman energy principle. The standard Lippmann-Schwinger equation is associated with kinematic uniform boundary conditions. As such, it features the Green operator of a finite body. The section therefore goes on with a discussion on the use of the Green operator of the infinite body in its stead. Then, an extension of the theorem of Eshelby (1957) is proposed, for ellipsoidal SVEs. The section closes with the so-called modified Lippmann-Schwinger equation, which results directly from this generalized theorem of Eshelby.

The new auxiliary problem with mixed boundary conditions is a typical example of elasticity problems in unbounded domains, the mathematical analysis of which is difficult (as opposed to elasticity problems in bounded domains). However, observing that the displacement is irrelevant for homogenization purposes (only the strain and stress are relevant), a sound but simple mathematical framework is set up in Sec. 4 to tackle such problems. This requires the definition of two pairs of orthogonal functional spaces: the spaces of divergence-free stresses and compatible strains on the one hand, and the spaces of statically admissible stresses and kinematically admissible strains on the other hand. These four spaces have the structure of Hilbert spaces. As a first application, a rigorous definition of the Green operator for strains of the unbounded domain is given. The section closes with the equivalence (for ellipsoidal SVEs) of the modified Lippmann-Schwinger equation and the auxiliary problem with mixed boundary conditions, which was stated without proof in Sec. 3.

In Sec. 5, we show that the classical principles of minimum potential and complementary energies can be extended to this new auxiliary problem. The well-posedness of the new auxiliary problem with mixed boundary conditions (as well as the equivalent modified Lippmann-Schwinger equation) results from these principles. Besides, it is shown that the minimum values of these energies are related to the newly defined apparent stiffness. This section closes with simple applications of these principles to prove the properties of the apparent stiffness previously listed in Sec. 2.

Finally, in Sec. 6 we derive an energy principle of the Hashin and Shtrikman (1962a) type. Such principle is useful for the numerical approximation of the modified Lippmann-Schwinger equation. Indeed, provided that the assumptions of this principle are verified, any approximation of the true polarization provides a bound on the apparent stiffness.

\section{Overview}

The present work is devoted to the homogenization of random heterogeneous, linearly elastic materials. The effective stiffness $\mathbf{C}^{\text {eff }}$ is usually computed as the limit of the apparent stiffness $\mathbf{C}^{\text {app }}$ of a bounded SVE $\Omega \subset \mathbb{R}^{d}$ as $|\Omega| \rightarrow+\infty$. In turn, the apparent stiffness $\mathbf{C}^{\text {app }}$ is defined from the solution to the following auxiliary problem: find the local displacement $\mathbf{u}$ such that

$$
\begin{array}{ll}
\mathbf{x} \in \Omega: & \nabla_{\mathbf{x}} \cdot \sigma=\mathbf{0}, \\
\mathbf{x} \in \Omega: & \boldsymbol{\sigma}(\mathbf{x})=\mathbf{C}(\mathbf{x}): \varepsilon(\mathbf{x}), \\
\mathbf{x} \in \Omega: & \boldsymbol{\varepsilon}(\mathbf{x})=\nabla_{\mathbf{x}}^{\mathrm{s}} \mathbf{u},
\end{array}
$$

where $\boldsymbol{\varepsilon}$ (resp. $\boldsymbol{\sigma}$ ) is the local strain (resp. stress) and $\mathbf{C}$ is the local stiffness of the heterogeneous material. Furthermore, $\nabla \cdot \sigma$ denotes the divergence of $\sigma$ and $\nabla^{\mathrm{s}} \mathbf{u}$ denotes the symmetric part of the gradient of $\mathbf{u}$; finally,":" stands for the double contraction on the nearest two indices. The above problem must be complemented with apropriate boundary conditions. Setting aside periodic boundary conditions, which will not be considered in the present paper, static or kinematic uniform boundary conditions are usually adopted (Hill, 1963, 1967; Mandel, 1972). 


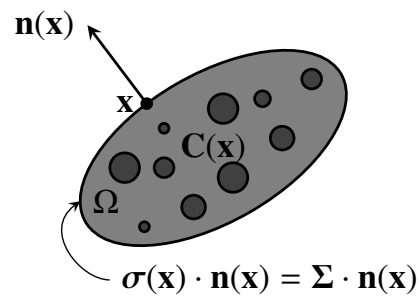

Problem SUBC

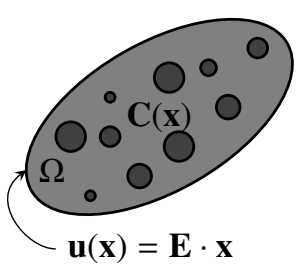

Problem KUBC

Figure 1: Graphical representation of the Problems SUBC (left) and KUBC (right).

In the case of static uniform boundary conditions, the loading parameter is the macroscopic stress $\boldsymbol{\Sigma}$, and the following tractions are specified at the boundary $\partial \Omega$ of the SVE

$$
\mathbf{x} \in \partial \Omega: \quad \boldsymbol{\sigma}(\mathbf{x}) \cdot \mathbf{n}(\mathbf{x})=\boldsymbol{\Sigma} \cdot \mathbf{n}(\mathbf{x}),
$$

where $\mathbf{n}$ is the outward normal to $\partial \Omega$. Owing to the linearity of the problem, the macroscopic strain $\bar{\varepsilon}$ (the volume average of the local strain) depends linearly on the macroscopic stress

$$
\bar{\varepsilon}=\mathbf{S}_{\mathrm{SUBC}}^{\mathrm{app}}: \mathbf{\Sigma}
$$

where $\mathbf{S}_{\text {SUBC }}^{\text {app }}$ is the apparent compliance. The apparent stiffness is then defined as $\mathbf{C}_{\text {SUBC }}^{\text {app }}=\left(\mathbf{S}_{\text {SUBC }}^{\text {app }}\right)^{-1}$. Together with the static uniform boundary conditions (2), Eqs. (1) define Problem SUBC (see Fig. 1, left).

In the case of kinematic uniform boundary conditions, the loading parameter is the macroscopic strain $\mathbf{E}$, and the following displacements are specified at the boundary $\partial \Omega$ of the SVE

$$
\mathbf{x} \in \partial \Omega: \quad \mathbf{u}(\mathbf{x})=\mathbf{E} \cdot \mathbf{x} .
$$

Owing to the linearity of the problem, the macroscopic stress $\bar{\sigma}$ (the volume average of the local strain) depends linearly on the macroscopic strain

$$
\overline{\boldsymbol{\sigma}}=\mathbf{C}_{\mathrm{KUBC}}^{\mathrm{app}}: \mathbf{E},
$$

where $\mathbf{C}_{\mathrm{KUBC}}^{\mathrm{app}}$ is the apparent stiffness. Together with the kinematic uniform boundary conditions (3), Eqs. (1) define Problem KUBC (see Fig. 1, right).

In the present paper, we propose a new auxiliary problem on the SVE $\Omega$, with mixed (both static and kinematic) boundary conditions. We call this new problem: Problem MBC. It requires the introduction of a homogeneous, linearly elastic reference medium $\mathbf{C}_{0}$, occupying the whole space $\mathbb{R}^{d}$, in which the SVE is embedded. Then, the local stiffness $\mathbf{C}$ and compliance $\mathbf{S}=\mathbf{C}^{-1}$, initially defined on $\Omega$ only, are extended to the whole space $\mathbb{R}^{d}$ as follows

$$
\mathbf{x} \in \mathbb{R}^{d} \backslash \Omega: \quad \mathbf{C}(\mathbf{x})=\mathbf{C}_{0}, \quad \mathbf{S}(\mathbf{x})=\mathbf{S}_{0},
$$

where $\mathbf{S}_{0}=\mathbf{C}_{0}^{-1}$ is the compliance of the reference medium. We assume that the reference medium is isotropic, with shear modulus $\mu_{0}$ and Poisson ratio $v_{0}$. Before we introduce Problem MBC, it should be noted that throughout this paper, overlined quantities denote volume averages over the bounded SVE $\Omega$, even if the quantity to be averaged is defined over the whole space $\mathbb{R}^{d}$

$$
\bar{a}=\frac{1}{|\Omega|} \int_{\Omega} a,
$$

and it is emphasized that the above quantity is meaningful, since the domain $\Omega$ is always bounded. 


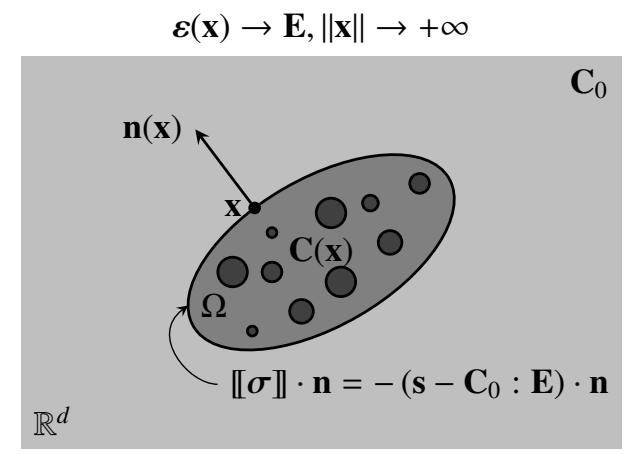

Figure 2: Graphical representation of Problem MBC. The SVE $\Omega$ is embedded in an infinite, homogeneous medium with stiffness $\mathbf{C}_{0}$, subjected to the strain $\mathbf{E}$ at infinity, and the force density $\left(\mathbf{s}-\mathbf{C}_{0}: \mathbf{E}\right) \cdot \mathbf{n}$ at $\partial \Omega$. The boundary condition at infinity $\boldsymbol{\varepsilon} \rightarrow \mathbf{E}$ should be understood as: $(\boldsymbol{\varepsilon}-\mathbf{E})$ has square-integrable components.

For the new, mixed boundary conditions introduced here, the loading parameter is again the macroscopic strain $\mathbf{E}$. In this problem, the unknowns are a constant, second-rank, symmetric tensor $\mathbf{s}$ and the local displacement $\mathbf{u}$, such that

$$
\begin{array}{ll}
\mathbf{x} \in \mathbb{R}^{d}: & \nabla_{\mathbf{x}} \cdot\left(\boldsymbol{\sigma}-\chi\left(\mathbf{s}-\mathbf{C}_{0}: \mathbf{E}\right)\right)=\mathbf{0}, \\
\mathbf{x} \in \mathbb{R}^{d}: & \boldsymbol{\sigma}(\mathbf{x})=\mathbf{C}(\mathbf{x}): \boldsymbol{\varepsilon}(\mathbf{x}), \\
\mathbf{x} \in \mathbb{R}^{d}: & \boldsymbol{\varepsilon}(\mathbf{x})=\nabla_{\mathbf{x}}^{\mathrm{s}} \mathbf{u}, \\
& \int_{\mathbb{R}^{d}}(\boldsymbol{\varepsilon}-\mathbf{E}): \mathbf{C}:(\boldsymbol{\varepsilon}-\mathbf{E})<+\infty, \\
& \overline{\boldsymbol{\varepsilon}}=\mathbf{E},
\end{array}
$$

where $\chi$ denotes the indicator function of the SVE $\Omega$.

Eq. (6d) means that the imposed strain at infinity is E. Since no boundary conditions are imposed to the displacement $\mathbf{u}$ in the above problem, it is determined up to a rigid body motion. From Eq. (6e), this imposed strain coincides with the macroscopic strain. Finally, Eq. (6a) means that $\sigma$ is in equilibrium with a surface force applied to $\partial \Omega$. Indeed, since $\left(\sigma-\chi\left(\mathbf{s}-\mathbf{C}_{0}: \mathbf{E}\right)\right)$ is divergence-free in $\mathbb{R}^{d}$, and $\chi\left(\mathbf{s}-\mathbf{C}_{0}: \mathbf{E}\right)$ is constant in $\Omega$ and $\mathbb{R}^{d} \backslash \Omega$, it is found that $\sigma$ is divergence-free in $\Omega$ and $\mathbb{R}^{d} \backslash \Omega$, and discontinuous across $\partial \Omega$. From the continuity of $\left(\sigma-\chi\left(\mathbf{s}-\mathbf{C}_{0}: \mathbf{E}\right)\right) \cdot \mathbf{n}$, we find

$$
\mathbf{x} \in \partial \Omega: \quad \llbracket \sigma \rrbracket(\mathbf{x}) \cdot \mathbf{n}(\mathbf{x})=-\left(\mathbf{s}-\mathbf{C}_{0}: \mathbf{E}\right) \cdot \mathbf{n}(\mathbf{x}),
$$

where $\mathbf{n}(\mathbf{x})$ is the outward normal to $\partial \Omega$ at $\mathbf{x}$ and $\llbracket \sigma \rrbracket(\mathbf{x})$ is the discontinuity of $\sigma$ at $\mathbf{x} \in \partial \Omega$, in the direction of $\mathbf{n}(\mathbf{x})$.

To sum up, Problem MBC corresponds to the elastic equilibrium of the heterogeneous SVE $\Omega$, embedded in the homogeneous, reference medium $\mathbf{C}_{0}$. The resulting infinite body is submitted to the imposed strain $\mathbf{E}$ at infinity, and the surface force density $\left(\mathbf{s}-\mathbf{C}_{0}: \mathbf{E}\right) \cdot \mathbf{n}$ applied to the boundary $\partial \Omega$, where the constant tensor $\mathbf{s}$ is chosen so as to ensure that $\bar{\varepsilon}=\mathbf{E}$ (see Fig. 2).

At first sight, Problem MBC might seem more complex than Problems SUBC and KUBC, due to the additional unknown s, which cannot in general be determined directly. However, it will be shown in Sec. 4.4 that for ellipsoidal SVEs, $\mathbf{s}$ is in fact equal to the macroscopic stress $(\mathbf{s}=\overline{\boldsymbol{\sigma}})$, and that Problem MBC is equivalent to the following modified Lippmann-Schwinger equation introduced in Sec. 3.4

$$
\left(\mathbf{C}-\mathbf{C}_{0}\right)^{-1}: \tau+\Gamma_{0}^{\infty} *(\tau-\chi \bar{\tau})=\mathbf{E}
$$

where the unknown polarization $\boldsymbol{\tau}$ is supported in $\Omega$, and $\boldsymbol{\Gamma}_{0}^{\infty}$ denotes the Green operator for strains of the unbounded reference medium (see Sec. 4.3). For matrix-inclusions composites, it is natural to select the matrix as reference medium. Then the polarization $\tau$ vanishes in the matrix, and Galerkin techniques can be used to discretize Eq. (8) with polarizations which are polynomial in each inclusion. The assembly of the underlying linear system is efficient because the Green operator $\Gamma_{0}^{\infty}$ is translation-invariant. The resulting numerical method will be reported elsewhere. 
The mathematical analysis of Problem MBC (in particular, its well-posedness) is greatly simplified by the principles of minimum potential and complementary energy introduced in Secs. 5.1 and 5.2, respectively. The potential and complementary energies $\mathcal{W}$ and $\mathcal{W}^{*}$ associated with Problem $\mathrm{MBC}$ are defined as follows

$$
\begin{aligned}
\mathcal{W}(\boldsymbol{\varepsilon}) & =\int_{\mathbb{R}^{d}}\left(\frac{1}{2} \boldsymbol{\varepsilon}: \mathbf{C}: \boldsymbol{\varepsilon}-\mathbf{E}: \mathbf{C}_{0}: \boldsymbol{\varepsilon}+\frac{1}{2} \mathbf{E}: \mathbf{C}_{0}: \mathbf{E}\right), \\
\mathcal{W}^{*}(\sigma) & =\int_{\mathbb{R}^{d}}\left(\frac{1}{2} \boldsymbol{\sigma}: \mathbf{S}: \boldsymbol{\sigma}-\mathbf{E}: \boldsymbol{\sigma}+\frac{1}{2} \mathbf{E}: \mathbf{C}_{0}: \mathbf{E}\right) .
\end{aligned}
$$

Theorem 8 states that the solution to Problem MBC minimizes the potential energy on the space of strains kinematically admissible with E. Similarly, Theorem 9 states that the solution to Problem MBC minimizes the complementary energy on the space of stresses statically admissible with E. Both spaces are defined in Sec. 4.2. The minimum potential energy principle can be invoked to prove that under the classical Assumption 1 below, Problem MBC is well-posed.

Assumption 1. There exists $\alpha>0$ and $\beta>0$ such that $\alpha \mathbf{I} \leq \mathbf{C} \leq \beta \mathbf{I}$ in $\Omega^{1}$.

Since $\mathbf{C}_{0}$ is positive definite, it is always possible to chose $0<\alpha<\alpha_{0}$ and $\beta>\beta_{0}$, where $\alpha_{0}>0$ (resp. $\beta_{0}$ ) is the smallest (resp. largest) eigenvalue of $\mathbf{C}_{0}$ (see Sec. 4.3). Therefore, we will assume in the remainder of this section that $\alpha \mathbf{I} \leq \mathbf{C} \leq \beta \mathbf{I}$ holds in the whole space $\mathbb{R}^{d}$.

For any value of the loading parameter $\mathbf{E}$ (which is equal to the macroscopic strain), the macroscopic stress $\bar{\sigma}$ is therefore well-defined, and depends linearly on $\mathbf{E}$, and it is possible to define the apparent stiffness $\mathbf{C}_{\mathrm{MBC}}^{\text {app }}\left(\mathbf{C}_{0}\right)$ associated with Problem MBC

$$
\overline{\boldsymbol{\sigma}}=\overline{\mathbf{C}: \boldsymbol{\varepsilon}}=\mathbf{C}_{\mathrm{MBC}}^{\mathrm{app}}\left(\mathbf{C}_{0}\right): \mathbf{E} .
$$

The apparent stiffness $\mathbf{C}_{\mathrm{MBC}}^{\mathrm{app}}\left(\mathbf{C}_{0}\right)$ thus defined corresponds to neither static uniform, nor kinematic uniform boundary conditions; in fact, it depends on the stiffness $\mathbf{C}_{0}$ of the reference medium. Its properties are listed below. It is shown in Sec. 5.1 that $\mathbf{C}_{\mathrm{MBC}}^{\mathrm{app}}$ is symmetric; furthermore, the following bounds hold (see Sec. 5.3)

$$
\mathbf{C}_{\mathrm{SUBC}}^{\mathrm{app}} \leq \mathbf{C}_{\mathrm{MBC}}^{\mathrm{app}}\left(\mathbf{C}_{0}\right) \leq \mathbf{C}_{\mathrm{KUBC}}^{\mathrm{app}}
$$

In turn, these inequalities have two important consequences. First, the apparent stiffness $\mathbf{C}_{\mathrm{MBC}}^{\text {app }}\left(\mathbf{C}_{0}\right)$ is positive definite (because of the positive-definiteness of $\mathbf{C}_{\mathrm{SUBC}}^{\mathrm{app}}$ ). Second, the present definition of the apparent stiffness is asymptotically consistent. Indeed, since

$$
\mathbf{C}_{\mathrm{SUBC}}^{\mathrm{app}}, \mathbf{C}_{\mathrm{KUBC}}^{\mathrm{app}} \rightarrow \mathbf{C}^{\mathrm{eff}} \text { as }|\Omega| \rightarrow+\infty,
$$

[under statistical homogeneity and ergodicity assumptions, see Sab (1992)], we also have

$$
\mathbf{C}_{\mathrm{MBC}}^{\mathrm{app}}\left(\mathbf{C}_{0}\right) \rightarrow \mathbf{C}^{\mathrm{eff}}
$$

Finally, the following limit cases established in Sec. 5.3 cast a new light on the classical static and kinematic uniform boundary conditions, which can now be considered as extreme cases of the more general mixed boundary conditions. More precisely

$$
\mathbf{C}_{\mathrm{MBC}}^{\mathrm{app}}\left(\mathbf{C}_{0}\right) \rightarrow \mathbf{C}_{\mathrm{SUBC}}^{\mathrm{app}} \text { when }\left\|\mathbf{C}_{0}\right\| \rightarrow 0 \quad \text { and } \quad \mathbf{C}_{\mathrm{MBC}}^{\mathrm{app}}\left(\mathbf{C}_{0}\right) \rightarrow \mathbf{C}_{\mathrm{KUBC}}^{\mathrm{app}} \text { when }\left\|\mathbf{C}_{0}\right\| \rightarrow+\infty .
$$

In other words, the new mixed boundary conditions introduced here "interpolate" between the classical static and kinematic uniform boundary conditions.

The paper closes with a third energy principle, of the Hashin and Shtrikman (1962a) type. Based on the modified Lippmann-Schwinger equation (8), the following functional is introduced in Sec. 6 for ellipsoidal SVEs

$$
\mathcal{H}_{\mathrm{MBC}}(\varpi)=\mathbf{E}: \bar{\varpi}-\frac{1}{2} \overline{\varpi:\left(\mathbf{C}-\mathbf{C}_{0}\right)^{-1}: \varpi}-\frac{1}{2} \overline{\varpi:\left(\Gamma_{0}^{\infty} *(\varpi-\chi \bar{\varpi})\right)} .
$$

\footnotetext{
${ }^{1}$ In the present paper, " $\mathbf{A} \leq \mathbf{B}$ " is to be understood in the sense of associated quadratic forms.
} 
The above expression is very similar to the classical functional of Hashin and Shtrikman (1962a). Furthermore, provided that the reference medium is stiffer (resp. softer) than all phases in the composite, this energy is minimum (resp. maximum) on the space of polarizations (see Theorem 12). This is a useful result for numerical applications, as it can be used to prove that numerical approximations of the apparent stiffness are actually bounds.

It should be noticed that Eq. (8) and (13) have already been introduced by Willis (1977), as a way to approximate the solution to Problem KUBC for infinitely large SVEs. Our approach is significantly different, as we intend to apply in future work the present developments to SVEs of finite size in a numerical setting. From this perspective, Eq. (8) is regarded as the Lippmann-Schwinger equation associated without approximation with Problem MBC (which differs from Problem KUBC), this equivalence being valid for ellipsoidal SVEs only.

To close this section, it is emphasized that the apparent stiffness defined through Problem MBC depends on the shape of the SVE $\Omega$, as indicated by the presence of the Hill tensor $\mathbf{P}_{\Omega}$ of $\Omega$ in Sec. 3.4. This is also true of apparent stifnesses defined through Problems SUBC and KUBC, and should therefore not come as a surprise. One of the main results of this paper is that, regardless of its exact shape, the apparent stiffness defined through Problem MBC converges to the uniquely defined effective stiffness as the size of the SVE $\Omega$ tends to infinity [under statistical homogeneity and ergodicity assumptions, see Sab (1992)]. In other words, the shape dependence vanishes for large SVEs.

Before we proceed with the proof of all the results stated in Sec. 2 above, we present in the following Sec. 3 the reasoning that led to the mixed boundary conditions introduced in this paper. The starting point is the LippmannSchwinger equation associated with Problem KUBC.

\section{The rationale behind the mixed boundary conditions}

\subsection{The standard Lippmann-Schwinger equation and the principle of Hashin and Shtrikman}

Introducing a homogeneous, linearly elastic reference medium $\mathbf{C}_{0}$, it is well known that Problem KUBC is equivalent to the Lippmann-Schwinger equation (Zeller and Dederichs, 1973; Kröner, 1977)

$$
\mathbf{x} \in \Omega: \quad \boldsymbol{\varepsilon}(\mathbf{x})+\left(\boldsymbol{\Gamma}_{0} \circledast\left(\left(\mathbf{C}-\mathbf{C}_{0}\right): \boldsymbol{\varepsilon}\right)\right)(\mathbf{x})=\mathbf{E},
$$

where the main unknown is the strain $\varepsilon$. In Eq. (14), $\boldsymbol{\Gamma}_{0}$ denotes the Green operator for strains of the bounded domain $\Omega$ with homogeneous stiffness $\mathbf{C}_{0}$, and ' $\circledast$ ' stands for the two-point product

$$
\left(\boldsymbol{\Gamma}_{0} \circledast \varpi\right)(\mathbf{x})=\int_{\mathbf{y} \in \Omega} \boldsymbol{\Gamma}_{0}(\mathbf{x}, \mathbf{y}): \varpi(\mathbf{y}) d \mathbf{y} .
$$

It is recalled that the Green operator for strains returns the (opposite of the) strain induced in the reference medium by the prestress $\varpi$. In other words, if $\mathbf{u}$ is the solution to the following problem

$$
\begin{aligned}
\mathbf{x} \in \Omega: & \nabla_{\mathbf{x}} \cdot \boldsymbol{\sigma}=\mathbf{0}, \\
\mathbf{x} \in \Omega: & \boldsymbol{\sigma}(\mathbf{x})=\mathbf{C}_{0}: \boldsymbol{\varepsilon}(\mathbf{x})+\varpi(\mathbf{x}), \\
\mathbf{x} \in \Omega: & \boldsymbol{\varepsilon}(\mathbf{x})=\nabla_{\mathbf{x}}^{\mathrm{s}} \mathbf{u}, \\
\mathbf{x} \in \partial \Omega: & \mathbf{u}(\mathbf{x})=\mathbf{0},
\end{aligned}
$$

then, by definition, $\boldsymbol{\Gamma}_{0} \circledast \boldsymbol{\varpi}=-\boldsymbol{\varepsilon}=-\nabla^{\mathrm{s}} \mathbf{u}$. Substituting in Eq. (14) the so-called polarization $\boldsymbol{\tau}(\mathbf{x})=\left(\mathbf{C}(\mathbf{x})-\mathbf{C}_{0}\right): \boldsymbol{\varepsilon}(\mathbf{x})$ to the strain $\boldsymbol{\varepsilon}(\mathbf{x})$, the Lippmann-Schwinger equation can be conveniently rewritten

$$
\mathbf{x} \in \Omega: \quad\left(\mathbf{C}(\mathbf{x})-\mathbf{C}_{0}\right)^{-1}: \tau(\mathbf{x})+\left(\boldsymbol{\Gamma}_{0} \circledast \tau\right)(\mathbf{x})=\mathbf{E},
$$

and the strain and stress are readily retrieved from the solution $\tau$ of equation (16)

$$
\mathbf{x} \in \Omega: \quad \boldsymbol{\varepsilon}(\mathbf{x})=\mathbf{E}-\left(\boldsymbol{\Gamma}_{0} \circledast \tau\right)(\mathbf{x}) \quad \text { and } \quad \boldsymbol{\sigma}(\mathbf{x})=\mathbf{C}_{0}: \boldsymbol{\varepsilon}(\mathbf{x})+\tau(\mathbf{x}) .
$$

Following the presentation of Willis (1977), the principle of Hashin and Shtrikman (1962a) can be seen as the variational counterpart of the Lippmann-Schwinger equation (16). Let $\tau$ denote the solution to this equation. Hashin and Shtrikman (1962a) first prove that $\tau$ is a critical point of the functional $\mathcal{H}_{\mathrm{KUBC}}$

$$
\mathcal{H}_{\mathrm{KUBC}}(\varpi)=\mathbf{E}: \bar{\varpi}-\frac{1}{2} \overline{\varpi:\left(\mathbf{C}-\mathbf{C}_{0}\right)^{-1}: \varpi}-\frac{1}{2} \overline{\varpi:\left(\boldsymbol{\Gamma}_{0} \circledast \varpi\right)} .
$$


Furthermore, the value of $\mathcal{H}_{\mathrm{KUBC}}$ at $\tau$ is known. Indeed, from Eq. (17)

$$
\mathcal{H}_{\mathrm{KUBC}}(\boldsymbol{\tau})=\frac{1}{2} \mathbf{E}: \overline{\boldsymbol{\sigma}}-\frac{1}{2} \mathbf{E}: \mathbf{C}_{0}: \overline{\boldsymbol{\varepsilon}}=\frac{1}{2} \mathbf{E}:\left(\mathbf{C}_{\mathrm{KUBC}}^{\mathrm{app}}-\mathbf{C}_{0}\right): \mathbf{E}
$$

Hashin and Shtrikman (1962a) then provide sufficient conditions for this critical point to be an extremum of $\mathcal{H}_{\mathrm{KUBC}}$. If the reference medium is stiffer (resp. softer) than the heterogeneous material, then $\mathcal{H}_{\mathrm{KUBC}}$ is minimum (resp. maximum) at $\tau$. In other words

1. if $\mathbf{C}(\mathbf{x}) \leq \mathbf{C}_{0}$ in $\Omega$, then $\tau$ is the unique minimizer of $\mathcal{H}_{\mathrm{KUBC}}$, and for all $\varpi$

$$
\frac{1}{2} \mathbf{E}: \mathbf{C}_{\mathrm{KUBC}}^{\mathrm{app}}: \mathbf{E} \leq \frac{1}{2} \mathbf{E}: \mathbf{C}_{0}: \mathbf{E}+\mathcal{H}_{\mathrm{KUBC}}(\varpi)
$$

2. if $\mathbf{C}(\mathbf{x}) \geq \mathbf{C}_{0}$ in $\Omega$, then $\tau$ is the unique maximizer of $\mathcal{H}_{\mathrm{KUBC}}$, and for all $\varpi$

$$
\frac{1}{2} \mathbf{E}: \mathbf{C}_{\mathrm{KUBC}}^{\mathrm{app}}: \mathbf{E} \geq \frac{1}{2} \mathbf{E}: \mathbf{C}_{0}: \mathbf{E}+\mathcal{H}_{\mathrm{KUBC}}(\varpi)
$$

The celebrated bounds of Hashin and Shtrikman (1962b) on the effective properties $\mathbf{C}^{\text {eff }}$ are retrieved under the assumption of statistical isotropy, with a phase-wise constant trial field $\varpi$.

\subsection{From Problem KUBC to Problem $\mathrm{KUBC}^{\infty}$}

The Lippmann-Schwinger equation (16) and the associated principle of Hashin and Shtrikman hold regardless of the shape of the bounded SVE $\Omega$. However, the Green operator for strains $\boldsymbol{\Gamma}_{0}$ is not translation-invariant if $\Omega$ is bounded. This makes the derivation of numerical methods based on Eq. (16) overly complicated, and of little practical value. By contrast, the Green operator for strains $\Gamma_{0}^{\infty}$ of the unbounded domain $\mathbb{R}^{d}$ is translation-invariant, and its closed-form expression for isotropic reference media is rather simple (see Appendix A). This has led many authors to substitute $\boldsymbol{\Gamma}_{0}^{\infty}$ to $\boldsymbol{\Gamma}_{0}$, assuming the approximation to be valid for domains $\Omega$ large enough. In other words, Eq. (16) is replaced with

$$
\mathbf{x} \in \Omega: \quad\left(\mathbf{C}(\mathbf{x})-\mathbf{C}_{0}\right)^{-1}: \tau(\mathbf{x})+\left(\boldsymbol{\Gamma}_{0}^{\infty} * \tau\right)(\mathbf{x})=\mathbf{E}^{\infty},
$$

and the associated elasticity problem will be called Problem $\mathrm{KUBC}^{\infty}$.

It is recalled that the Green operator $\Gamma_{0}^{\infty}$ of the unbounded domain $\mathbb{R}^{d}$ returns the (opposite of the) strain $\varepsilon$ induced by the prestress $\varpi$. In other words, for any prestress $\varpi, \varepsilon=-\Gamma_{0}^{\infty} * \varpi$ is the unique compatible strain such that $\mathbf{C}_{0}: \boldsymbol{\varepsilon}+\varpi$ is divergence-free. A more formal definition of $\boldsymbol{\Gamma}_{0}^{\infty}$ (including requirements on the regularity of $\varpi$ ) will be given in Sec. 4.3 .

Eq. (20) is specified in $\Omega$ only. However, $\mathbf{E}^{\infty}-\Gamma_{0}^{\infty} * \boldsymbol{\tau}$ defines a strain field $\boldsymbol{\varepsilon}$ in the whole space $\mathbb{R}^{d}$. Similarly, if $\boldsymbol{\tau}$ is extended to $\mathbf{0}$ in $\mathbb{R}^{d} \backslash \Omega$, then $\sigma=\mathbf{C}_{0}: \boldsymbol{\varepsilon}+\boldsymbol{\tau}$ is a divergence-free stress field defined in the whole space $\mathbb{R}^{d}$. Having thus extended $\sigma$ and $\varepsilon$, it is seen from the definition of $\Gamma_{0}^{\infty}$ that Problem $\mathrm{KUBC}^{\infty}$ corresponds to the elastic equilibrium of the SVE $\Omega$, embedded in an infinite, homogeneous body with elastic stiffness $\mathbf{C}_{0}$, and subjected to a uniform strain $\mathbf{E}^{\infty}$ at infinity (see Fig. 3).

From Problem $\mathrm{KUBC}^{\infty}$, it is possible to define a new apparent stiffness $\mathbf{C}_{\mathrm{KUBC}}^{\text {app }}$ of the finite body $\Omega$. The derivation must account for the fact that the loading parameter $\mathbf{E}^{\infty}$ is not the average strain over the domain $\Omega$. Therefore, both the average stress $\bar{\sigma}$ and strain $\bar{\varepsilon}$ must be computed from the local stress $\sigma$ and $\varepsilon$. Because of the linearity of Eq. (20) with respect to $\mathbf{E}^{\infty}$, both $\bar{\sigma}$ and $\overline{\boldsymbol{\varepsilon}}$ depend linearly on the strain at infinity $\mathbf{E}^{\infty}$

$$
\overline{\boldsymbol{\varepsilon}}=\mathbf{A}_{\mathrm{KUBC}^{\infty}}: \mathbf{E}^{\infty}, \quad \overline{\boldsymbol{\sigma}}=\mathbf{B}_{\mathrm{KUBC}^{\infty}}: \mathbf{E}^{\infty},
$$

where $\mathbf{A}_{\mathrm{KUBC}}{ }^{\infty}$ and $\mathbf{B}_{\mathrm{KUBC}}{ }^{\infty}$ are fourth-rank localization tensors. The apparent stiffness results from the elimination of the loading parameter

$$
\mathbf{C}_{\mathrm{KUBC}}^{\mathrm{app}}=\mathbf{B}_{\mathrm{KUBC}^{\infty}}: \mathbf{A}_{\mathrm{KUBC}^{\infty}}^{-1}
$$

This approach has been successfully used by e.g. Torquato (1997) for theoretical series expansions of the effective stiffness, and by Fond et al. $(2001,2002)$ for numerical computations of these quantities. It can also be related to Maxwell's far-field methodology (McCartney and Kelly, 2008; McCartney, 2010). 


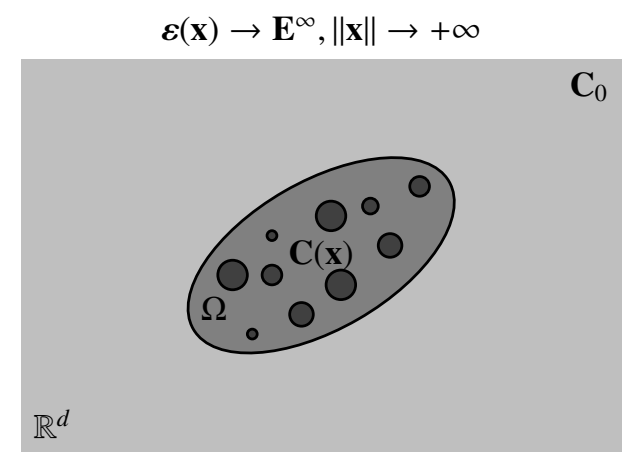

Figure 3: Graphical representation of Problem $\mathrm{KUBC}^{\infty}$. The SVE $\Omega$ is embedded in an infinite, homogeneous medium with stiffness $\mathbf{C}_{0}$, subjected to the strain $\mathbf{E}^{\infty}$ at infinity. The boundary condition at infinity $\boldsymbol{\varepsilon} \rightarrow \mathbf{E}^{\infty}$ should be understood as: $\left(\boldsymbol{\varepsilon}-\mathbf{E}^{\infty}\right)$ has square-integrable components.

To close this section, it should be emphasized that Eq. (20) is not equivalent to Eq. (16); in other words, $\mathbf{C}_{\mathrm{KUBC}}^{\mathrm{app}} \neq \mathbf{C}_{\mathrm{KUBC}}^{\mathrm{app}}$. Using Saint-Venant's principle, Willis (1977) proved that substituting $\boldsymbol{\Gamma}_{0}^{\infty}$ to $\boldsymbol{\Gamma}_{0}$ is valid in the limit of infinitely large SVEs (provided that only the fluctuations of $\tau$ are considered). However, this substitution is no longer valid in the case of finite-size SVEs.

Moreover, adoption of Eq. (20) leads to the loss of the principle of Hashin and Shtrikman. Indeed, if we were to define the functional $\mathcal{H}_{\mathrm{KUBC}^{\infty}}$ as follows

$$
\mathcal{H}_{\mathrm{KUBC}^{\infty}}(\varpi)=\mathbf{E}^{\infty}: \bar{\varpi}-\frac{1}{2} \overline{\varpi:\left(\mathbf{C}-\mathbf{C}_{0}\right)^{-1}: \varpi}-\frac{1}{2} \overline{\varpi:\left(\mathbf{\Gamma}_{0}^{\infty} * \varpi\right)},
$$

then we would readily find that the solution $\tau$ to Eq. (20) is a critical point of $\mathcal{H}_{\mathrm{KUBC}}$. However, there is no longer a direct relationship between the value of $\mathcal{H}_{\mathrm{KUBC}}{ }^{\infty}$ at $\tau$ and the apparent stiffness $\mathbf{C}_{\mathrm{KUBC}}^{\mathrm{app}}$; in other words, Eq. (19) does not generalize to $\mathcal{H}_{\mathrm{KUBC}}{ }^{\infty}$ and $\mathbf{C}_{\mathrm{KUBC}}^{\mathrm{app}}$. Therefore, it would not be possible to resort to the principle of Hashin and Shtrikman to exhibit bounds on $\mathbf{C}_{\mathrm{KUBC}}^{\mathrm{app}}$.

In section 3.4 below, we introduce the so-called modified Lippmann-Schwinger equation (8) as a way to address all these shortcomings. We show that this new equation is also based on the substitution of $\boldsymbol{\Gamma}_{0}$ with $\boldsymbol{\Gamma}_{0}^{\infty}$. Like Willis (1977), we apply the Green operator of the infinite body to the fluctuations of $\tau$ only; however, we do not regard the solution to the modified Lippmann-Schwinger equation as an approximate solution to the initial Problem KUBC. Instead, we show in Sec. 4.4 that the modified Lippmann-Schwinger equation (23) is strictly equivalent to Problem MBC (which differs from Problem KUBC).

Although it is based on a similar substitution, Eq. (23) is superior to Eq. (20) in that the loading parameter is the macroscopic strain. As a consequence, Eq. (19) does generalize to $\mathcal{H}_{\mathrm{MBC}}$ and $\mathbf{C}_{\mathrm{MBC}}^{\text {app }}$, and it is possible to exhibit bounds on the apparent stiffness $\mathbf{C}_{\mathrm{MBC}}^{\mathrm{app}}$.

\subsection{A generalization of Eshelby's theorem}

The motivation for the modified Lippmann-Schwinger equation introduced in Sec. 3.4 comes from Theorem 2 below, which can be seen as an extension of Eshelby's theorem (Eshelby, 1957). Eshelby's theorem states that, in an ellipsoidal inclusion ${ }^{2}$, the strain caused by a uniform prestress is uniform. For the sake of completeness, this result is recalled without proof in a compact form in Theorem 1.

Theorem 1 (Eshelby, 1957). If $\Omega$ is ellipsoidal, then for any constant prestress $\varpi$

$$
\mathbf{x} \in \Omega: \quad\left(\boldsymbol{\Gamma}_{0}^{\infty} *(\chi \varpi)\right)(\mathbf{x})=\mathbf{P}_{\Omega}: \varpi,
$$

where $\mathbf{P}_{\Omega}$ denotes the Hill tensor of $\Omega$ with respect to the reference medium $\mathbf{C}_{0}$.

\footnotetext{
${ }^{2}$ Following Eshelby (1957), "inclusion" should be understood here as a prestressed, bounded region in a homogeneous, infinite medium.
} 
Using the same notations as in Theorem 1, Theorem 2 relates the average of the strain to the average of the (heterogeneous) prestress in an ellipsoidal inclusion.

Theorem 2. If $\Omega$ is ellipsoidal

$$
\overline{\Gamma_{0}^{\infty} * \varpi}=\mathbf{P}_{\Omega}: \bar{\varpi},
$$

for any prestress $\varpi$ with square-integrable components supported in $\Omega$.

In the above theorem, the assumption that $\varpi$ is supported in the ellipsoidal domain $\Omega$ is essential. Furthermore, it is recalled that, although $\Gamma_{0}^{\infty} * \varpi$ is defined in the whole space $\mathbb{R}^{d}$, only its values in $\Omega$ are considered to compute the volume average $\overline{\Gamma_{0}^{\infty} * \varpi}$ [see Eq. (5)]. A proof of Theorem 2, based on Fubini's theorem, can be found in Rodin and Hwang (1991) (see Property 1). In the present paper, we propose an alternative proof, based on Lemma 1, which can be seen as a generalization of the Maxwell-Betti reciprocal work theorem (see Appendix B).

\subsection{The modified Lippmann-Schwinger equation}

The derivation of the modified Lippmann-Schwinger equation (8) relies on the fact that the SVE $\Omega$ is ellipsoidal, so that Theorem 2 applies. Let $\tau$ be the solution to Eq. (20); introducing the strain $\varepsilon=\mathbf{E}^{\infty}-\Gamma_{0}^{\infty} * \tau$, we have $\overline{\boldsymbol{\varepsilon}}=\mathbf{E}^{\infty}-\mathbf{P}_{\Omega}: \bar{\tau}$. Since the volume average $\overline{\boldsymbol{\varepsilon}}$ is by definition the macroscopic strain $\mathbf{E}$, Eq. (20) also reads

$$
\mathbf{x} \in \Omega: \quad\left(\mathbf{C}(\mathbf{x})-\mathbf{C}_{0}\right)^{-1}: \tau(\mathbf{x})+\left(\boldsymbol{\Gamma}_{0}^{\infty} * \boldsymbol{\tau}\right)(\mathbf{x})-\mathbf{P}_{\Omega}: \bar{\tau}=\mathbf{E} .
$$

From Theorem 1, we have

$$
\mathbf{x} \in \Omega: \quad \mathbf{P}_{\Omega}: \overline{\boldsymbol{\tau}}=\left(\boldsymbol{\Gamma}_{0}^{\infty} *(\chi \overline{\boldsymbol{\tau}})\right)(\mathbf{x}),
$$

and upon substitution into Eq. (21)

$$
\mathbf{x} \in \Omega: \quad\left(\mathbf{C}(\mathbf{x})-\mathbf{C}_{0}\right)^{-1}: \boldsymbol{\tau}(\mathbf{x})+\left(\boldsymbol{\Gamma}_{0}^{\infty} *(\boldsymbol{\tau}-\chi \bar{\tau})\right)(\mathbf{x})=\mathbf{E} .
$$

The main asset of Eq. (23), which is equivalent to Eq. (20), lies in the fact that the loading parameter is now the macroscopic strain $\mathbf{E}$, instead of the auxiliary strain $\mathbf{E}^{\infty}$. This leads us to introduce the so-called modified LippmannSchwinger equation, with unknown $\tau$ supported in $\Omega$. This equation has already been introduced in Sec. 2 [see Eq. (8)]; for the sake of completeness, it is recalled below

$$
\left(\mathbf{C}-\mathbf{C}_{0}\right)^{-1}: \tau+\Gamma_{0}^{\infty} *(\tau-\chi \bar{\tau})=\mathbf{E}
$$

The above analysis shows that the solution $\tau$ to Eq. (8) is also the solution to Eq. (20), with $\mathbf{E}^{\infty}=\mathbf{E}+\mathbf{P}_{\Omega}: \overline{\boldsymbol{\tau}}$

$$
\mathbf{x} \in \Omega: \quad \mathbf{E}^{\infty}-\left(\boldsymbol{\Gamma}_{0}^{\infty} * \boldsymbol{\tau}\right)(\mathbf{x})=\mathbf{E}-\left(\boldsymbol{\Gamma}_{0}^{\infty} *(\boldsymbol{\tau}-\chi \overline{\boldsymbol{\tau}})\right)(\mathbf{x})
$$

The left-hand side is the strain defined in $\mathbb{R}^{d}$ by the solution to Eq. (20), while the right-hand side is the strain defined in $\mathbb{R}^{d}$ by the solution to Eq. (8). The above identity shows that both strains are equal inside $\Omega$. However, because Eq. (22) is valid in $\Omega$ only, this identity does not hold outside $\Omega$.

This means that the strain $\left(\mathbf{E}-\Gamma_{0}^{\infty} *(\boldsymbol{\tau}-\chi \overline{\boldsymbol{\tau}})\right)$ defined by the solution to the modified Lippmann-Schwinger equation (8) is not the solution to Problem KUBC ${ }^{\infty}$ defined in Sec. 3.2. It will be shown in Sec. 4.4 below that it is the solution to Problem MBC, introduced in Sec. 2. More precisely, the modified Lippmann-Schwinger equation (8) is equivalent to Problem MBC for ellipsoidal SVEs.

The modified Lippmann-Schwinger equation is a typical example of elasticity problems in unbounded domains (the whole space $\mathbb{R}^{d}$ in the present case). Their mathematical analysis (namely, existence and uniqueness of the solution) is notoriously more involved than that of problems posed in bounded domains. However, observing that the displacement is irrelevant for homogenization purposes (only the strain and stress are relevant), a mathematical framework is set up in Sec. 4 below to tackle such problems. As an application, a rigorous definition of the Green operator $\boldsymbol{\Gamma}_{0}^{\infty}$ is provided. It is then possible to prove that (for ellipsoidal SVEs), Problem MBC is equivalent to the modified Lippmann-Schwinger equation (8). 


\section{A mathematical framework for elasticity problems in unbounded domains}

Regularity requirements will be necessary to ensure existence and uniqueness of the solution to the problems considered below. A natural choice is related to the finiteness of the elastic energy carried by stresses and strains. We therefore introduce the space $\mathbb{L}^{2}\left(\mathbb{R}^{d}\right)$ of second-rank, symmetric tensors with square-integrable components in $\mathbb{R}^{d}$. It is recalled that $\mathbb{L}^{2}\left(\mathbb{R}^{d}\right)$ is a Hilbert space for the scalar product

$$
\langle\sigma, \varepsilon\rangle=\int_{\mathbb{R}^{d}} \sigma: \varepsilon,
$$

and the associated norm will be denoted $\|\cdot\|_{2}$. Similarly, for $\Omega \subset \mathbb{R}^{d}$, we introduce the space $\mathbb{L}^{2}(\Omega)$ of second-rank, symmetric tensors with square-integrable components in $\Omega$. In the remainder of this paper, any element of $\mathbb{L}^{2}(\Omega)$ will be considered as an element of $\mathbb{L}^{2}\left(\mathbb{R}^{d}\right)$, supported in $\Omega$.

It should be noted that all stresses and strains arising in this paper are not finite-energy stresses and strains. Indeed, we will frequently meet situations where $\sigma \sim \sigma^{\infty}$ (resp. $\left.\boldsymbol{\varepsilon} \sim \boldsymbol{\varepsilon}^{\infty}\right)$ when $\|\mathbf{x}\| \rightarrow+\infty\left(\sigma^{\infty}, \boldsymbol{\varepsilon}^{\infty}\right.$ are constant second-rank, symmetric tensors). We will then require that $\sigma-\sigma^{\infty} \in \mathbb{L}^{2}\left(\mathbb{R}^{d}\right)\left[\right.$ resp. $\left.\boldsymbol{\varepsilon}-\boldsymbol{\varepsilon}^{\infty} \in \mathbb{L}^{2}\left(\mathbb{R}^{d}\right)\right]$.

\subsection{Divergence-free and compatible tensors}

In the remainder of this paper, the usual definition of divergence-free tensors is adopted. The second-rank, symmetric tensor $\boldsymbol{\sigma}$ is said to be divergence-free $(\nabla \cdot \sigma=\mathbf{0})$ in $\mathbb{R}^{d}$ if for any vector $\mathbf{u}$ with $\mathrm{C}_{0}^{\infty}\left(\mathbb{R}^{d}\right)$ components, $\left\langle\boldsymbol{\sigma}, \nabla^{\mathrm{s}} \mathbf{u}\right\rangle=0$, where the notation $\langle\boldsymbol{\sigma}, \boldsymbol{\varepsilon}\rangle=\left\langle\sigma_{i j}, \varepsilon_{i j}\right\rangle$ stands for the application of the test function $\boldsymbol{\varepsilon}$ to the distribution $\boldsymbol{\sigma}$. If $\boldsymbol{\sigma} \in \mathbb{L}^{2}\left(\mathbb{R}^{d}\right)$, this notation coincides with the usual scalar product over $\mathbb{L}^{2}\left(\mathbb{R}^{d}\right)$ [as defined in Eq. (24)]. We then define the space $\mathbb{S}$ of divergence-free tensors with square-integrable components

$$
\mathbb{S}=\left\{\boldsymbol{\sigma} \in \mathbb{L}^{2}\left(\mathbb{R}^{d}\right) \text { such that } \nabla \cdot \boldsymbol{\sigma}=\mathbf{0}\right\} .
$$

Clearly, $\mathbb{S}$ is a closed subspace of the Hilbert space $\mathbb{L}^{2}\left(\mathbb{R}^{d}\right)$ (since strong convergence implies weak convergence). Therefore, it is a Hilbert space.

It is well-known that Poincaré's and Korn's inequalities do not apply to unbounded domains [although some progress has been made in this direction, see e.g. Kondrat'ev and Oleinik (1988) and Amrouche et al. (2012)]. As a consequence, existence and uniqueness of the displacement is difficult to prove for elasticity problems set in such geometries. By constrast, proving the existence and uniqueness of the strain is relatively easy, as will be shown below. The corresponding displacement $\mathbf{u}$ is unique up to a rigid body motion; besides if $\boldsymbol{\varepsilon}$ has square-integrable components, then $\mathbf{u}$ has $\mathrm{H}_{\mathrm{loc}}^{1}\left(\mathbb{R}^{d}\right.$ ) components (Amrouche et al., 2012). While this limitation could be prohibitive for some applications, it is of little consequence for homogenization purposes, where the quantities of interest are the stress $\sigma$ and strain $\varepsilon$.

This observation led us to adopt a very lax definition of compatible strains: $\varepsilon$ is said to be compatible if there exists a distribution vector field $\mathbf{u}$ such that $\boldsymbol{\varepsilon}=\nabla^{\mathrm{s}} \mathbf{u}$ in the sense of distributions. We then define the space $\mathbb{K}$ of compatible tensors with square-integrable components

$$
\mathbb{K}=\left\{\boldsymbol{\varepsilon} \in \mathbb{L}^{2}\left(\mathbb{R}^{d}\right) \text { such that there exists } \mathbf{u} \in \mathcal{D}^{\prime}\left(\mathbb{R}^{d}\right)^{d}, \boldsymbol{\varepsilon}=\nabla^{\mathrm{s}} \mathbf{u}\right\},
$$

where $\mathcal{D}^{\prime}\left(\mathbb{R}^{d}\right)$ denotes the space of distributions in $\mathbb{R}^{d}$. Moreau's characterization of compatible strains (see Theorem 3 below) proves that $\mathbb{K}$ is a closed subspace of $\mathbb{L}^{2}\left(\mathbb{R}^{d}\right)$. Therefore, it is a Hilbert space; this will allow us to invoke the Lax-Milgram theorem in Sec. 4.3.

Theorem 3 (Moreau, 1979). Let $\varepsilon$ be a second-rank, symmetric tensor with components in $\mathcal{D}^{\prime}\left(\mathbb{R}^{d}\right)$; for the existence of a vector $\mathbf{u}$ with components in $\mathcal{D}^{\prime}\left(\mathbb{R}^{d}\right)$, such that $\varepsilon=\nabla^{\mathrm{s}} \mathbf{u}$, it is necessary and sufficient that for any divergence-free tensor $\boldsymbol{\sigma}$ with $\mathrm{C}_{0}^{\infty}\left(\mathbb{R}^{d}\right)$ components, $\langle\boldsymbol{\varepsilon}, \boldsymbol{\sigma}\rangle=0$.

Theorem 3 ensures the existence of $\mathbf{u}$, with no guarantee on its regularity, since $\nabla^{\mathrm{s}}$ is to be understood in the sense of distributions [although some extensions to this theorem have recently been proposed by Amrouche et al. (2006)].

We close this section with a theorem which is essential for the remainder of this paper. It should be noted that orthogonal decompositions similar to that stated below have already been considered by other authors [see e.g. Milton (2002) in the case of periodic boundary conditions]. 
Theorem 4. The space $\mathbb{L}^{2}\left(\mathbb{R}^{d}\right)$ of symmetric, square-integrable tensors is the direct orthogonal sum of the space $\mathbb{S}$ of divergence-free tensors and $\mathbb{K}$ of compatible tensors

$$
\mathbb{L}^{2}\left(\mathbb{R}^{d}\right)=\stackrel{\perp}{\oplus} \mathbb{K} .
$$

Proof of Theorem 4. Since $\mathbb{K}$ is a closed subspace of $\mathbb{L}^{2}\left(\mathbb{R}^{d}\right)$, we have

$$
\mathbb{L}^{2}\left(\mathbb{R}^{d}\right)=\mathbb{K} \stackrel{\perp}{\oplus} \mathbb{K}^{\perp},
$$

and it suffices to prove that $\mathbb{K}^{\perp}=\mathbb{S}$. We first consider $\sigma \in \mathbb{S}$ and $\boldsymbol{\varepsilon} \in \mathbb{K}$, and show that $\langle\boldsymbol{\sigma}, \boldsymbol{\varepsilon}\rangle=0$ (which proves that $\mathbb{S} \subset \mathbb{K}^{\perp}$ ). By construction, $\sigma$ and $\varepsilon$ have square-integrable components, and their Fourier transforms $\hat{\sigma}$ and $\hat{\varepsilon}$ also have square-integrable components. Furthermore the compatible strain $\boldsymbol{\varepsilon}$ satisfies the following classical compatibility conditions

$$
\partial_{h l}^{2} \varepsilon_{i j}+\partial_{i j}^{2} \varepsilon_{h l}-\partial_{j l}^{2} \varepsilon_{i h}-\partial_{i h}^{2} \varepsilon_{j l}=0,
$$

which are to be understood in the sense of distributions. The above identity reads in Fourier space

$$
k_{h} k_{l} \hat{\varepsilon}_{i j}(\mathbf{k})+k_{i} k_{j} \hat{\varepsilon}_{h l}(\mathbf{k})-k_{j} k_{l} \hat{\varepsilon}_{i h}(\mathbf{k})-k_{i} k_{h} \hat{\varepsilon}_{j l}(\mathbf{k})=0 .
$$
identity

Multiplying by $\delta_{i h} \hat{\sigma}_{j l}^{*}(\mathbf{k})$ and using Einstein's convention for repeated indices leads in intrinsic form to the following

$$
\hat{\sigma}^{*}(\mathbf{k}): \hat{\varepsilon}(\mathbf{k})=2 \mathbf{n} \cdot \hat{\boldsymbol{\sigma}}^{*}(\mathbf{k}) \cdot \hat{\boldsymbol{\varepsilon}}(\mathbf{k}) \cdot \mathbf{n}-\left(\mathbf{n} \cdot \hat{\boldsymbol{\sigma}}^{*}(\mathbf{k}) \cdot \mathbf{n}\right)(\mathbf{n} \cdot \hat{\boldsymbol{\varepsilon}}(\mathbf{k}) \cdot \mathbf{n}),
$$

where $\mathbf{n}=\|\mathbf{k}\|^{-1} \mathbf{k}$. Now, $\mathbf{n} \cdot \hat{\boldsymbol{\sigma}}(\mathbf{k})=\mathbf{0}$, since $\nabla \cdot \boldsymbol{\sigma}=\mathbf{0}$. As a result, Eq. (25) reduces to $\hat{\boldsymbol{\sigma}}^{*}(\mathbf{k}): \hat{\boldsymbol{\varepsilon}}(\mathbf{k})=0$. Then, using Plancherel's theorem $\langle\boldsymbol{\sigma}, \boldsymbol{\varepsilon}\rangle=(2 \pi)^{-d}\left\langle\hat{\boldsymbol{\sigma}}^{*}, \hat{\boldsymbol{\varepsilon}}\right\rangle=0$.

Conversely, let $\sigma \in \mathbb{K}^{\perp}$; then, for all $\boldsymbol{\varepsilon} \in \mathbb{K},\langle\boldsymbol{\sigma}, \boldsymbol{\varepsilon}\rangle=0$. In particular, for all vector $\mathbf{u}$ with $\mathrm{C}_{0}^{\infty}\left(\mathbb{R}^{d}\right)$ components, we have $\left\langle\boldsymbol{\sigma}, \nabla^{\mathrm{s}} \mathbf{u}\right\rangle=0$. By definition of the divergence, $\nabla \cdot \boldsymbol{\sigma}=\mathbf{0}$; in other words, $\boldsymbol{\sigma} \in \mathbb{S}$, and $\mathbb{K}^{\perp} \subset \mathbb{S}$. As a conclusion, $\mathbb{K}^{\perp}=\mathbb{S}$, and the proof is complete.

\subsection{Statically admissible stresses and kinematically admissible strains}

In view of Problem MBC [see Eqs. (6)], it will prove convenient to introduce the following two subspaces of $\mathbb{L}^{2}\left(\mathbb{R}^{d}\right)$. First, $\mathbb{S}^{\dagger}$ is defined as the space of stress tensors $\sigma$ such that $\sigma-\chi \mathbf{s}$ is divergence-free for a constant, symmetric tensor $\mathbf{s}$; then, $\mathbb{K}^{\dagger}$ is defined as the space of compatible strains with null volume average over $\Omega$

$$
\begin{aligned}
\mathbb{S}^{\dagger} & =\left\{\boldsymbol{\sigma} \in \mathbb{L}^{2}\left(\mathbb{R}^{d}\right) \text { such that there exists } \mathbf{s} \text { constant, symmetric, } \boldsymbol{\sigma}-\chi \mathbf{s} \in \mathbb{S}\right\}, \\
\mathbb{K}^{\dagger} & =\{\boldsymbol{\varepsilon} \in \mathbb{K} \text {, such that } \overline{\boldsymbol{\varepsilon}}=\mathbf{0}\} .
\end{aligned}
$$

We further define statically admissible stresses and kinematically admissible strains as follows. The stress $\sigma$ is said to be statically admissible with the macroscopic strain $\mathbf{E}$ if $\sigma \in \mathbf{C}_{0}: \mathbf{E}+\mathbb{S}^{\dagger}$. Similarly, the strain $\varepsilon$ is said to be kinematically admissible with the macroscopic strain $\mathbf{E}$ if $\boldsymbol{\varepsilon} \in \mathbf{E}+\mathbb{K}^{\dagger}$.

It can readily be verified that $\mathbb{S}^{\dagger}$ is a closed subspace of the Hilbert space $\mathbb{L}^{2}\left(\mathbb{R}^{d}\right)$. Similarly, $\mathbb{K}^{\dagger}$ is also a closed subspace of the Hilbert space $\mathbb{K}$. Therefore, both $\mathbb{S}^{\dagger}$ and $\mathbb{K}^{\dagger}$ are Hilbert spaces [for the $\mathbb{L}^{2}\left(\mathbb{R}^{d}\right)$ norm]. Furthermore, the following orthogonal decomposition holds.

Theorem 5. The space $\mathbb{L}^{2}\left(\mathbb{R}^{d}\right)$ is the direct orthogonal sum of $\mathbb{S}^{\dagger}$ and $\mathbb{K}^{\dagger}$

$$
\mathbb{L}^{2}\left(\mathbb{R}^{d}\right)=\mathbb{S}^{\dagger} \stackrel{\perp}{\oplus} \mathbb{K}^{\dagger}
$$

Proof of Theorem 5. Since $\mathbb{S}^{\dagger}$ is a closed subspace of $\mathbb{L}^{2}\left(\mathbb{R}^{d}\right)$, we have

$$
\mathbb{L}^{2}\left(\mathbb{R}^{d}\right)=\mathbb{S}^{\dagger} \stackrel{\perp}{\oplus}\left(\mathbb{S}^{\dagger}\right)^{\perp}
$$


and it suffices to prove that $\left(\mathbb{S}^{\dagger}\right)^{\perp}=\mathbb{K}^{\dagger}$. We first consider $\boldsymbol{\sigma} \in \mathbb{S}^{\dagger}$ and $\boldsymbol{\varepsilon} \in \mathbb{K}^{\dagger}$, and show that $\langle\boldsymbol{\sigma}, \boldsymbol{\varepsilon}\rangle=0$ (which proves that $\left.\mathbb{K}^{\dagger} \subset\left(\mathbb{S}^{\dagger}\right)^{\perp}\right)$. By definition, there exists $\mathbf{s}$ such that $(\sigma-\chi \mathbf{s}) \in \mathbb{S}$, and

$$
\langle\boldsymbol{\sigma}, \boldsymbol{\varepsilon}\rangle=\langle\boldsymbol{\sigma}-\chi \mathbf{s}, \boldsymbol{\varepsilon}\rangle+\langle\chi \mathbf{s}, \boldsymbol{\varepsilon}\rangle=\langle\boldsymbol{\sigma}-\chi \mathbf{s}, \boldsymbol{\varepsilon}\rangle+|\Omega| \mathbf{s}: \overline{\boldsymbol{\varepsilon}} .
$$

The first term vanishes from Theorem $4[(\boldsymbol{\sigma}-\chi \mathbf{s}) \in \mathbb{S}$, and $\boldsymbol{\varepsilon} \in \mathbb{K}]$. The second term also vanishes, since $\overline{\boldsymbol{\varepsilon}}=\mathbf{0}$. Therefore, $\langle\boldsymbol{\sigma}, \boldsymbol{\varepsilon}\rangle=0$ and $\mathbb{K}^{\dagger} \subset\left(\mathbb{S}^{\dagger}\right)^{\perp}$.

Conversely, we now consider $\varepsilon \in\left(\mathbb{S}^{\dagger}\right)^{\perp}$. Then for all $\boldsymbol{\sigma} \in \mathbb{S}^{\dagger},\langle\boldsymbol{\sigma}, \boldsymbol{\varepsilon}\rangle=0$. In particular, since $\mathbb{S} \subset \mathbb{S}^{\dagger}$, for all $\sigma \in \mathbb{S},\langle\boldsymbol{\sigma}, \boldsymbol{\varepsilon}\rangle=0$. From Theorem 3, this means that $\boldsymbol{\varepsilon} \in \mathbb{K}$. Similarly, for all constant, symmetric tensors $\mathbf{s}$, $0=\langle\chi \mathbf{s}, \boldsymbol{\varepsilon}\rangle=|\Omega| \mathbf{s}: \overline{\boldsymbol{\varepsilon}}$, and $\overline{\boldsymbol{\varepsilon}}=\mathbf{0}$. Therefore $\boldsymbol{\varepsilon} \in \mathbb{K}^{\dagger}$, and $\left(\mathbb{S}^{\dagger}\right)^{\perp} \subset \mathbb{K}^{\dagger}$.

\subsection{A mathematical definition of the Green operator $\boldsymbol{\Gamma}_{0}^{\infty}$}

It is now possible to give a precise definition of the Green operator $\boldsymbol{\Gamma}_{0}^{\infty}$ associated to the reference medium with stiffness $\mathbf{C}_{0}$. Since $\mathbf{C}_{0}$ is positive definite, we have $\alpha_{0} \mathbf{I} \leq \mathbf{C}_{0} \leq \beta_{0} \mathbf{I}$, where $\alpha_{0}>0$ (resp. $\beta_{0}$ ) is the smallest (resp. largest) eigenvalue of $\mathbf{C}_{0}$, and $\mathbf{I}$ is the fourth-rank identity tensor. For $\varpi \in \mathbb{L}^{2}\left(\mathbb{R}^{d}\right)$, we then consider the following problem

Find $\boldsymbol{\varepsilon} \in \mathbb{K}$ such that $\left(\mathbf{C}_{0}: \boldsymbol{\varepsilon}+\varpi\right) \in \mathbb{S}$.

In order to prove that this problem is well-posed, we first show that it is equivalent to minimizing the potential energy $\mathcal{W}_{0}(\boldsymbol{\varepsilon})=\frac{1}{2}\left\langle\boldsymbol{\varepsilon}, \mathbf{C}_{0}: \boldsymbol{\varepsilon}\right\rangle+\langle\boldsymbol{\varpi}, \boldsymbol{\varepsilon}\rangle$, which is well-defined in $\mathbb{K}$.

Theorem 6. $\varepsilon \in \mathbb{K}$ solves $E q$. (26) if, and only if, $\varepsilon$ minimizes the potential energy $\mathcal{W}_{0}$.

Proof of theorem 6. We first consider a solution $\boldsymbol{\varepsilon} \in \mathbb{K}$ to Eq. (26). Then, for all $\delta \boldsymbol{\varepsilon} \in \mathbb{K}$,

$$
\mathcal{W}_{0}(\boldsymbol{\varepsilon}+\delta \boldsymbol{\varepsilon})-\mathcal{W}_{0}(\boldsymbol{\varepsilon})=\frac{1}{2}\left\langle\delta \boldsymbol{\varepsilon}, \mathbf{C}_{0}: \delta \boldsymbol{\varepsilon}\right\rangle+\left\langle\mathbf{C}_{0}: \boldsymbol{\varepsilon}+\varpi, \delta \boldsymbol{\varepsilon}\right\rangle,
$$

and the second term vanishes from Eq. (26) and Theorem 4. Since the first term is positive, we find that $\mathcal{W}_{0}$ is minimum at $\boldsymbol{\varepsilon}$.

Conversely, let $\boldsymbol{\varepsilon}$ be a minimizer of $\mathcal{W}_{0}$. Then $\mathcal{W}_{0}$ is stationary at $\boldsymbol{\varepsilon}$, and for all $\delta \boldsymbol{\varepsilon} \in \mathbb{K},\left\langle\mathbf{C}_{0}: \boldsymbol{\varepsilon}+\boldsymbol{\varpi}, \delta \boldsymbol{\varepsilon}\right\rangle=0$. In other words, $\left(\mathbf{C}_{0}: \varepsilon+\varpi\right) \in \mathbb{K}^{\perp}$ and, from Theorem $4,\left(\mathbf{C}_{0}: \varepsilon+\varpi\right) \in \mathbb{S}$, which proves that $\varepsilon$ is a solution to Eq. (26).

Theorem 7. The potential energy $\mathcal{W}_{0}$ has a unique minimizer on $\mathbb{K}$.

Proof of theorem 7. We first note that for all $\boldsymbol{\varepsilon} \in \mathbb{K}, \mathcal{W}_{0}(\boldsymbol{\varepsilon})=\frac{1}{2} \mathcal{A}_{0}(\boldsymbol{\varepsilon}, \boldsymbol{\varepsilon})+\langle\boldsymbol{\sigma}, \boldsymbol{\varepsilon}\rangle$, where $\mathcal{A}_{0}\left(\boldsymbol{\varepsilon}_{1}, \boldsymbol{\varepsilon}_{2}\right)=\left\langle\boldsymbol{\varepsilon}_{1}, \mathbf{C}_{0}: \boldsymbol{\varepsilon}_{2}\right\rangle$. Minimizing $\mathcal{W}_{0}$ therefore reduces to the following variational problem

Find $\boldsymbol{\varepsilon} \in \mathbb{K}$ such that $\mathcal{A}_{0}(\boldsymbol{\varepsilon}, \delta \boldsymbol{\varepsilon})+\langle\boldsymbol{\varpi}, \delta \boldsymbol{\varepsilon}\rangle$ for all $\delta \boldsymbol{\varepsilon} \in \mathbb{K}$.

From the Cauchy-Schwarz inequality, the linear form $\delta \boldsymbol{\varepsilon} \mapsto\langle\boldsymbol{\varpi}, \delta \boldsymbol{\varepsilon}\rangle$ is continuous over $\mathbb{K}$. Then, from $\alpha_{0} \mathbf{I} \leq \mathbf{C}_{0} \leq$ $\beta_{0} \mathbf{I}, \mathcal{A}_{0}$ is continuous and coercive over $\mathbb{K}$. Since $\mathbb{K}$ is a Hilbert space, the Lax-Milgram theorem applies, and $\mathcal{W}_{0}$ has a unique minimizer $\boldsymbol{\varepsilon} \in \mathbb{K}$.

For any prestress $\varpi \in \mathbb{L}^{2}\left(\mathbb{R}^{d}\right)$, we then define $\Gamma_{0}^{\infty}(\varpi)=-\boldsymbol{\varepsilon}$, where $\boldsymbol{\varepsilon} \in \mathbb{K}$ is the unique solution to Eq. (26). $\boldsymbol{\Gamma}_{0}^{\infty}$ thus defined is obviously a linear operator from $\mathbb{L}^{2}\left(\mathbb{R}^{d}\right)$ to $\mathbb{K}$. Furthermore, it is continuous. Indeed, using $\boldsymbol{\varepsilon}=-\boldsymbol{\Gamma}_{0}^{\infty}(\boldsymbol{\varpi})$ as a test function in Eq. (27), it is found from the Cauchy-Schwarz inequality

$$
\alpha_{0}\|\boldsymbol{\varepsilon}\|_{2}^{2} \leq\left\langle\boldsymbol{\varepsilon}, \mathbf{C}_{0}: \boldsymbol{\varepsilon}\right\rangle=-\langle\boldsymbol{\varpi}, \boldsymbol{\varepsilon}\rangle \leq\|\varpi\|_{2}\|\boldsymbol{\varepsilon}\|_{2} .
$$

In other words, $\left\|\boldsymbol{\Gamma}_{0}^{\infty}(\varpi)\right\|_{2} \leq \frac{1}{\alpha_{0}}\|\boldsymbol{\varpi}\|_{2}$. Finally, $\boldsymbol{\Gamma}_{0}^{\infty}$ is obviously invariant by translation; therefore the mapping of $\Gamma_{0}^{\infty}$ to $\varpi$ should be understood as a convolution product: $\Gamma_{0}^{\infty}(\varpi)=\Gamma_{0}^{\infty} * \varpi$.

With this definition of the Green operator $\boldsymbol{\Gamma}_{0}^{\infty}$, it is readily verified that an equivalent formulation of Problem $\mathrm{KUBC}^{\infty}$ [see Eq. (20)] is

Find $\varepsilon \in \mathbf{E}^{\infty}+\mathbb{K}$ such that $\sigma \in \mathbf{C}_{0}: \mathbf{E}^{\infty}+\mathbb{S}$, where $\sigma=\mathbf{C}: \boldsymbol{\varepsilon}$. 
Similarly, an equivalent formulation of Problem MBC [see Eq. (6)] is

Find $\boldsymbol{\varepsilon} \in \mathbf{E}+\mathbb{K}^{\dagger}$ such that $\sigma \in \mathbf{C}_{0}: \mathbf{E}+\mathbb{S}^{\dagger}$, where $\sigma=\mathbf{C}: \boldsymbol{\varepsilon}$.

Finally, we note that if $\Omega$ is ellipsoidal and $\varpi \in \mathbb{L}^{2}(\Omega)$, then $\boldsymbol{\varepsilon} \in \mathbb{K}^{\dagger}$ and $\boldsymbol{\sigma} \in \mathbb{S}^{\dagger}$, where $\boldsymbol{\varepsilon}=-\boldsymbol{\Gamma}_{0}^{\infty} *(\varpi-\chi \overline{\boldsymbol{\varpi}})$ and $\sigma=\mathbf{C}_{0}: \boldsymbol{\varepsilon}+\varpi$ (attention must be drawn to the fact that $\varpi$ is supported in $\Omega$ ).

\subsection{Equivalence between the modified Lippmann-Schwinger equation and Problem $\mathrm{MBC}$}

In the present section, we show that the modified Lippmann-Schwinger equation (8) introduced in Sec. 3.4 is equivalent to Problem MBC [see Eq. (28)] when the SVE $\Omega$ is ellipsoidal.

We first show that any solution to the modified Lippmann-Schwinger equation provides a solution to Problem MBC. Let $\tau$ be a solution to Eq. (8). We then define in the whole space $\mathbb{R}^{d}$ the local strain $\varepsilon$ as follows

$$
\varepsilon=\mathbf{E}-\Gamma_{0}^{\infty} *(\boldsymbol{\tau}-\chi \overline{\boldsymbol{\tau}})
$$

From the definition of the Green operator for strains $\boldsymbol{\Gamma}_{0}^{\infty}$ [see Eq. (26)], $\boldsymbol{\varepsilon} \in \mathbf{E}+\mathbb{K}$. Furthermore, $\boldsymbol{\tau}$ is supported in the ellipsoidal domain $\Omega$, and Theorem 2 applies, leading to $\overline{\boldsymbol{\varepsilon}}=\mathbf{E}$; in other words, $\boldsymbol{\varepsilon} \in \mathbf{E}+\mathbb{K}^{\dagger}$.

Defining in $\mathbb{R}^{d}$ the local stress $\sigma=\mathbf{C}: \boldsymbol{\varepsilon}$, it is found from the combination of Eqs. (4), (8) and (29) that $\sigma=\mathbf{C}_{0}: \boldsymbol{\varepsilon}+\boldsymbol{\tau}$. Using again the definition of the Green operator for strains $\boldsymbol{\Gamma}_{0}^{\infty}$ [see Eq. (26)],

$$
\boldsymbol{\sigma}-\mathbf{C}_{0}: \mathbf{E}-\chi \overline{\boldsymbol{\tau}}=\mathbf{C}_{0}:(\boldsymbol{\varepsilon}-\mathbf{E})+\boldsymbol{\tau}-\chi \overline{\boldsymbol{\tau}} \in \mathbb{S},
$$

and $\boldsymbol{\sigma} \in \mathbf{C}_{0}: \mathbf{E}+\mathbb{S}^{\dagger}$ (with $\mathbf{s}=\overline{\boldsymbol{\tau}}$ ). Therefore, $\boldsymbol{\varepsilon}$ defined by Eq. (29) is such that $\boldsymbol{\varepsilon} \in \mathbf{E}+\mathbb{K}^{\dagger}$ and $\mathbf{C}: \boldsymbol{\varepsilon} \in \mathbf{C}_{0}: \mathbf{E}+\mathbb{S}^{\dagger} ; \boldsymbol{\varepsilon}$ is a solution to Problem MBC [see Eq. (28)].

Conversely, let $\boldsymbol{\varepsilon} \in \mathbf{E}+\mathbb{K}^{\dagger}$ be a solution to Problem MBC, and $\boldsymbol{\sigma}=\mathbf{C}: \boldsymbol{\varepsilon}$. Since $\sigma \in \mathbf{C}_{0}: \mathbf{E}+\mathbb{S}^{\dagger}$, there exists a constant, second-rank, symmetric tensor s such that

$$
\sigma^{\prime}=\sigma-\mathbf{C}_{0}: \mathbf{E}-\chi\left(\mathbf{s}-\mathbf{C}_{0}: \mathbf{E}\right) \in \mathbb{S}
$$

further introducing $\boldsymbol{\varepsilon}^{\prime}=\boldsymbol{\varepsilon}-\mathbf{E} \in \mathbb{K}^{\dagger}$ and $\boldsymbol{\tau}=\left(\mathbf{C}-\mathbf{C}_{0}\right): \boldsymbol{\varepsilon}$, it is readily verified that

$$
\boldsymbol{\sigma}^{\prime}=\mathbf{C}_{0}: \boldsymbol{\varepsilon}^{\prime}+\boldsymbol{\tau}-\chi\left(\mathbf{s}-\mathbf{C}_{0}: \mathbf{E}\right),
$$

and, from the definition of $\boldsymbol{\Gamma}_{0}^{\infty}$ [see Eq. (26)]

$$
\boldsymbol{\varepsilon}^{\prime}=-\boldsymbol{\Gamma}_{0}^{\infty} *\left(\boldsymbol{\tau}-\chi\left(\mathbf{s}-\mathbf{C}_{0}: \mathbf{E}\right)\right) .
$$

Theorem 2 applies to $\left(\boldsymbol{\tau}-\chi\left(\mathbf{s}-\mathbf{C}_{0}: \mathbf{E}\right)\right)$ which is supported in the ellipsoidal SVE $\Omega$, and $\overline{\boldsymbol{\varepsilon}^{\prime}}=\mathbf{P}_{\Omega}:\left(\overline{\boldsymbol{\tau}}-\mathbf{s}+\mathbf{C}_{0}: \mathbf{E}\right)$. This volume average is null, since $\boldsymbol{\varepsilon}^{\prime} \in \mathbb{K}^{\dagger}$, which implies that $\overline{\boldsymbol{\tau}}=\mathbf{s}-\mathbf{C}_{0}: \mathbf{E}$, and $\boldsymbol{\varepsilon}=\mathbf{E}-\boldsymbol{\Gamma}_{0}^{\infty} *(\boldsymbol{\tau}-\chi \overline{\boldsymbol{\tau}})$; in other words, $\boldsymbol{\tau}$ is a solution to Eq. (8). Furthermore, $\mathbf{s}=\overline{\boldsymbol{\tau}}+\mathbf{C}_{0}: \mathbf{E}=\overline{\boldsymbol{\sigma}}$.

As a conclusion, the modified Lippmann-Schwinger equation (8) is an equivalent formulation of Problem MBC when the SVE $\Omega$ is ellipsoidal.

In Sec. 5 below, we show that it is possible to state minimum potential (see Sec. 5.1) and complementary (see Sec. 5.2) energy principles associated with Problem MBC [see Eq. (28)]. The relevant expressions of these energies [see Eqs. (9) and (10)] are analogous to the classical expressions, with corrections ensuring convergence of the integrals over $\mathbb{R}^{d}$. Furthermore, the stationary value of both these energies is related to the apparent stiffness $\mathbf{C}_{\mathrm{MBC}}^{\text {app }}\left(\mathbf{C}_{0}\right)$ [see Eqs. (34) and (38)].

\section{Energy principles associated with Problem MBC}

\subsection{The principle of minimum potential energy}

For $\boldsymbol{\varepsilon} \in \mathbf{E}+\mathbb{K}^{\dagger}$, the potential energy $\mathcal{W}(\boldsymbol{\varepsilon})$ of the heterogeneous material is defined by Eq. (9). It can readily be verified that this integral is well defined over $\mathbf{E}+\mathbb{K}^{\dagger}$. Indeed, its integrand is equal to

$$
\frac{1}{2} \boldsymbol{\varepsilon}^{\prime}: \mathbf{C}: \boldsymbol{\varepsilon}^{\prime}+\mathbf{E}:\left(\mathbf{C}-\mathbf{C}_{0}\right): \boldsymbol{\varepsilon}^{\prime}+\frac{1}{2} \mathbf{E}:\left(\mathbf{C}-\mathbf{C}_{0}\right): \mathbf{E},
$$


where $\boldsymbol{\varepsilon}^{\prime}=\boldsymbol{\varepsilon}-\mathbf{E}$. In the above expression, the last two terms vanish outside $\Omega$, while the first term is integrable over $\mathbb{R}^{d}$ by definition of $\mathbb{K}^{\dagger}$. Therefore, an alternative expression of $\mathcal{W}(\boldsymbol{\varepsilon})$ is

$$
\mathcal{W}(\boldsymbol{\varepsilon})=\frac{1}{2}\left\langle\boldsymbol{\varepsilon}^{\prime}, \mathbf{C}: \boldsymbol{\varepsilon}^{\prime}\right\rangle+|\Omega| \mathbf{E}: \overline{\left(\mathbf{C}-\mathbf{C}_{0}\right): \boldsymbol{\varepsilon}^{\prime}}+\frac{1}{2}|\Omega| \mathbf{E}:\left(\overline{\mathbf{C}}-\mathbf{C}_{0}\right): \mathbf{E}
$$

In the remainder of this section, we will seek the minimum of the potential energy over the space of kinematically admissible strains. We first note that optimizing $\boldsymbol{\varepsilon} \mapsto \mathcal{W}(\boldsymbol{\varepsilon})$ on $\mathbf{E}+\mathbb{K}^{\dagger}$ is equivalent to optimizing $\boldsymbol{\varepsilon}^{\prime} \mapsto \mathcal{W}\left(\boldsymbol{\varepsilon}^{\prime}+\mathbf{E}\right)$ on $\mathbb{K}^{\dagger}$. Then, from Eq. (30)

$$
\mathcal{W}\left(\boldsymbol{\varepsilon}^{\prime}+\mathbf{E}\right)-\frac{1}{2}|\Omega| \mathbf{E}:\left(\overline{\mathbf{C}}-\mathbf{C}_{0}\right): \mathbf{E}=\frac{1}{2} \mathcal{A}\left(\boldsymbol{\varepsilon}^{\prime}, \boldsymbol{\varepsilon}^{\prime}\right)+|\Omega| \mathbf{E}: \overline{\left(\mathbf{C}-\mathbf{C}_{0}\right): \boldsymbol{\varepsilon}^{\prime}},
$$

where $\mathcal{A}$ is a bilinear form over $\mathbb{K}^{\dagger} \times \mathbb{K}^{\dagger}$ defined as follows

$$
\mathcal{A}\left(\boldsymbol{\varepsilon}_{1}^{\prime}, \boldsymbol{\varepsilon}_{2}^{\prime}\right)=\left\langle\boldsymbol{\varepsilon}_{1}^{\prime}, \mathbf{C}: \boldsymbol{\varepsilon}_{2}^{\prime}\right\rangle,
$$

and optimizing $\mathcal{W}$ over $\mathbf{E}+\mathbb{K}^{\dagger}$ reduces to solving the following variational problem

Find $\boldsymbol{\varepsilon}^{\prime} \in \mathbb{K}^{\dagger}$ such that $\mathcal{A}\left(\boldsymbol{\varepsilon}^{\prime}, \delta \boldsymbol{\varepsilon}\right)+|\Omega| \mathbf{E}: \overline{\left(\mathbf{C}-\mathbf{C}_{0}\right): \delta \boldsymbol{\varepsilon}}=0$ for all $\delta \boldsymbol{\varepsilon} \in \mathbb{K}^{\dagger}$,

the corresponding optimizer of $\mathcal{W}$ being $\boldsymbol{\varepsilon}^{\prime}+\mathbf{E}$. It can readily be verified that, under Assumption 1 , the linear form $\boldsymbol{\varepsilon}^{\prime} \mapsto|\Omega| \mathbf{E}: \overline{\left(\mathbf{C}-\mathbf{C}_{0}\right): \boldsymbol{\varepsilon}^{\prime}}$ is continuous, while the bilinear form $\mathcal{A}$ is continuous and coercive. Therefore, the Lax-Milgram theorem applies, and the potential energy $\mathcal{W}$ has a unique minimizer on $\mathbf{E}+\mathbb{K}^{\dagger}$.

Theorem 8 below characterizes the solutions of Problem MBC in terms of minimums of the potential energy. As such, it generalizes to unbounded domains the classical principle of minimum potential energy; its proof can be found in Appendix C.

Theorem 8 (Principle of minimum potential energy). $\boldsymbol{\varepsilon} \in \mathbf{E}+\mathbb{K}^{\dagger}$ solves Problem $\mathrm{MBC}$ if, and only if, $\boldsymbol{\varepsilon}$ minimizes the potential energy $\mathcal{W}$ on $\mathbf{E}+\mathbb{K}^{\dagger}$.

The above results prove that Problem MBC has a unique solution in $\mathbf{E}+\mathbb{K}^{\dagger}$. As a consequence, when $\Omega$ is ellipsoidal, the equivalent modified Lippmann-Schwinger equation (8) also has a unique solution in $\mathbb{L}^{2}(\Omega)$.

Since Problem MBC is well-posed, the apparent stiffness $\mathbf{C}_{\mathrm{MBC}}^{\mathrm{app}}\left(\mathbf{C}_{0}\right)$ is well-defined. It can further be shown that the minimum value of $\mathcal{W}$ is related to the apparent stiffness. Indeed, let $\boldsymbol{\varepsilon}^{\prime} \in \mathbb{K}^{\dagger}$ be the unique minimizer of $\mathcal{W}\left(\mathbf{E}+\boldsymbol{\varepsilon}^{\prime}\right)$, and $\boldsymbol{\varepsilon}=\boldsymbol{\varepsilon}^{\prime}+\mathbf{E}$. Then, from Eqs. (11) and (33)

$$
\mathcal{A}\left(\boldsymbol{\varepsilon}^{\prime}, \boldsymbol{\varepsilon}^{\prime}\right)=-|\Omega| \mathbf{E}: \overline{\left(\mathbf{C}-\mathbf{C}_{0}\right): \boldsymbol{\varepsilon}^{\prime}}=-|\Omega| \mathbf{E}:\left(\mathbf{C}_{\mathrm{MBC}}^{\mathrm{app}}\left(\mathbf{C}_{0}\right)-\overline{\mathbf{C}}\right): \mathbf{E},
$$

and, using (31)

$$
\mathcal{W}(\boldsymbol{\varepsilon})=\frac{1}{2}|\Omega| \mathbf{E}: \overline{\left(\mathbf{C}-\mathbf{C}_{0}\right): \boldsymbol{\varepsilon}^{\prime}}+\frac{1}{2}|\Omega| \mathbf{E}:\left(\overline{\mathbf{C}}-\mathbf{C}_{0}\right): \mathbf{E}=\frac{1}{2}|\Omega| \mathbf{E}:\left(\mathbf{C}_{\mathrm{MBC}}^{\mathrm{app}}\left(\mathbf{C}_{0}\right)-\mathbf{C}_{0}\right): \mathbf{E} .
$$

In other words

$$
\min _{\boldsymbol{\varepsilon} \in \mathbf{E}+\mathbb{K}^{\dagger}} \mathcal{W}(\boldsymbol{\varepsilon})=\frac{1}{2}|\Omega| \mathbf{E}:\left(\mathbf{C}_{\mathrm{MBC}}^{\mathrm{app}}\left(\mathbf{C}_{0}\right)-\mathbf{C}_{0}\right): \mathbf{E} .
$$

To close this section, we further show that the apparent stiffness $\mathbf{C}_{\mathrm{MBC}}^{\mathrm{app}}\left(\mathbf{C}_{0}\right)$ is symmetric. To this end, we consider two macroscopic strains $\mathbf{E}_{1}$ and $\mathbf{E}_{2}$, and $\boldsymbol{\varepsilon}_{i}^{\prime} \in \mathbb{K}^{\dagger}$ the unique minimizer of $\mathcal{W}\left(\mathbf{E}_{i}+\boldsymbol{\varepsilon}_{i}^{\prime}\right)(i=1,2)$. Then, using again Eqs. (11) and (33)

$$
\begin{aligned}
& \mathcal{A}\left(\boldsymbol{\varepsilon}_{1}^{\prime}, \boldsymbol{\varepsilon}_{2}^{\prime}\right)=-|\Omega| \mathbf{E}_{1}: \overline{\left(\mathbf{C}-\mathbf{C}_{0}\right):\left(\boldsymbol{\varepsilon}_{2}^{\prime}-\mathbf{E}_{2}\right)}=-|\Omega| \mathbf{E}_{1}:\left(\mathbf{C}_{\mathrm{MBC}}^{\mathrm{app}}\left(\mathbf{C}_{0}\right)-\overline{\mathbf{C}}\right): \mathbf{E}_{2}, \\
& \mathcal{A}\left(\boldsymbol{\varepsilon}_{2}^{\prime}, \boldsymbol{\varepsilon}_{1}^{\prime}\right)=-|\Omega| \mathbf{E}_{2}: \overline{\left(\mathbf{C}-\mathbf{C}_{0}\right):\left(\boldsymbol{\varepsilon}_{1}^{\prime}-\mathbf{E}_{1}\right)}=-|\Omega| \mathbf{E}_{2}:\left(\mathbf{C}_{\mathrm{MBC}}^{\mathrm{app}}\left(\mathbf{C}_{0}\right)-\overline{\mathbf{C}}\right): \mathbf{E}_{1},
\end{aligned}
$$

and

$$
\mathbf{E}_{1}: \mathbf{C}_{\mathrm{MBC}}^{\mathrm{app}}\left(\mathbf{C}_{0}\right): \mathbf{E}_{2}=\mathbf{E}_{2}: \mathbf{C}_{\mathrm{MBC}}^{\mathrm{app}}\left(\mathbf{C}_{0}\right): \mathbf{E}_{1},
$$

since the bilinear form $\mathcal{A}$ is symmetric. Therefore, the apparent stiffness is symmetric. 


\subsection{The principle of minimum complementary energy}

For $\sigma \in \mathbf{C}_{0}: \mathbf{E}+\mathbb{S}^{\dagger}$, the complementary energy $\mathcal{W}^{*}(\sigma)$ of the heterogeneous material is defined by Eq. (10). It can readily be verified that this integral is well defined over $\mathbf{C}_{0}: \mathbf{E}+\mathbb{S}^{\dagger}$. Indeed, its integrand is equal to

$$
\frac{1}{2} \boldsymbol{\sigma}^{\prime}: \mathbf{S}: \boldsymbol{\sigma}^{\prime}+\mathbf{E}:\left(\mathbf{C}_{0}: \mathbf{S}-\mathbf{I}\right): \boldsymbol{\sigma}^{\prime}+\frac{1}{2} \mathbf{E}:\left(\mathbf{C}_{0}: \mathbf{S}: \mathbf{C}_{0}-\mathbf{C}_{0}\right): \mathbf{E},
$$

where $\sigma^{\prime}=\sigma-\mathbf{C}_{0}: \mathbf{E}$. In the above expression, the last two terms are null outside $\Omega$, while the first term is integrable over $\mathbb{R}^{d}$ by definition of $\mathbb{S}^{\dagger}$. Therefore, an alternative expression of $\mathcal{W}^{*}(\sigma)$ is

$$
\mathcal{W}^{*}(\boldsymbol{\sigma})=\frac{1}{2}\left\langle\boldsymbol{\sigma}^{\prime}, \mathbf{S}: \boldsymbol{\sigma}^{\prime}\right\rangle+|\Omega| \mathbf{E}: \overline{\left(\mathbf{C}_{0}: \mathbf{S}-\mathbf{I}\right): \boldsymbol{\sigma}^{\prime}}+\frac{1}{2}|\Omega| \mathbf{E}:\left(\mathbf{C}_{0}: \overline{\mathbf{S}}: \mathbf{C}_{0}-\mathbf{C}_{0}\right): \mathbf{E} .
$$

In the remainder of this section, we will seek the minimum of the complementary energy over the space of statically admissible stresses. We first note that optimizing $\sigma \mapsto W^{*}(\sigma)$ on $\mathbf{C}_{0}: \mathbf{E}+\mathbb{S}^{\dagger}$ is equivalent to optimizing $\sigma^{\prime} \mapsto \mathcal{W}^{*}\left(\sigma^{\prime}+\mathbf{C}_{0}: \mathbf{E}\right)$ on $\mathbb{S}^{\dagger}$. Then, from Eq. (35)

$$
\mathcal{W}^{*}\left(\boldsymbol{\sigma}^{\prime}+\mathbf{C}_{0}: \mathbf{E}\right)-\frac{1}{2}|\Omega| \mathbf{E}:\left(\mathbf{C}_{0}: \overline{\mathbf{S}}: \mathbf{C}_{0}-\mathbf{C}_{0}\right): \mathbf{E}=\frac{1}{2} \mathcal{A}^{*}\left(\boldsymbol{\sigma}^{\prime}, \boldsymbol{\sigma}^{\prime}\right)+|\Omega| \mathbf{E}: \overline{\left(\mathbf{C}_{0}: \mathbf{S}-\mathbf{I}\right): \boldsymbol{\sigma}^{\prime}},
$$

where $\mathcal{A}^{*}$ is a bilinear form over $\mathbb{S}^{\dagger} \times \mathbb{S}^{\dagger}$ defined as follows

$$
\mathcal{A}^{*}\left(\sigma_{1}^{\prime}, \sigma_{2}^{\prime}\right)=\left\langle\sigma_{1}^{\prime}, \mathbf{S}: \sigma_{2}^{\prime}\right\rangle,
$$

and optimizing $\mathcal{W}^{*}$ over $\mathbf{C}_{0}: \mathbf{E}+\mathbb{S}^{\dagger}$ reduces to solving the following variational problem

$$
\text { Find } \boldsymbol{\sigma}^{\prime} \in \mathbb{S}^{\dagger} \text { such that } \mathcal{A}^{*}\left(\boldsymbol{\sigma}^{\prime}, \delta \boldsymbol{\sigma}\right)+|\Omega| \mathbf{E}: \overline{\left(\mathbf{C}_{0}: \mathbf{S}-\mathbf{I}\right): \delta \boldsymbol{\sigma}}=0 \text { for all } \delta \boldsymbol{\sigma} \in \mathbb{S}^{\dagger} \text {, }
$$

the corresponding optimizer of $\mathcal{W}^{*}$ being $\sigma^{\prime}+\mathbf{C}_{0}: \mathbf{E}$. It can readily be verified that, under Assumption 1, the linear form $\sigma^{\prime} \mapsto|\Omega| \mathbf{E}: \overline{\left(\mathbf{C}_{0}: \mathbf{S}-\mathbf{I}\right): \boldsymbol{\sigma}^{\prime}}$ is continuous, while the bilinear form $\mathcal{A}^{*}$ is continuous and coercive. Therefore, the Lax-Milgram theorem applies, and the complementary energy $\mathcal{W}^{*}$ has a unique minimizer on $\mathbf{C}_{0}: \mathbf{E}+\mathbb{S}^{\dagger}$.

Theorem 9 below characterizes the solutions of Problem MBC in terms of minimums of the complementary energy. As such, it generalizes to unbounded domains the classical principle of minimum complementary energy; its proof can be found in Appendix C.

Theorem 9 (Principle of minimum complementary energy). $\sigma \in \mathbf{C}_{0}: \mathbf{E}+\mathbb{S}^{\dagger}$ solves Problem MBC if, and only if, $\boldsymbol{\sigma}$ minimizes the complementary energy $\mathcal{W}^{*}$ on $\mathbf{C}_{0}: \mathbf{E}+\mathbb{S}^{\dagger}$.

It can further be shown that the minimum value of $\mathcal{W}^{*}$ is related to the apparent stiffness $\mathbf{C}_{\mathrm{MBC}}^{\mathrm{app}}\left(\mathbf{C}_{0}\right)$. Indeed, let $\sigma^{\prime} \in \mathbb{S}^{\dagger}$ be the unique minimizer of $\mathcal{W}^{*}\left(\mathbf{C}_{0}: \mathbf{E}+\sigma^{\prime}\right)$. Then, from Eqs. (11) and (37)

$$
\mathcal{A}^{*}\left(\boldsymbol{\sigma}^{\prime}, \boldsymbol{\sigma}^{\prime}\right)=-|\Omega| \mathbf{E}: \overline{\left(\mathbf{C}_{0}: \mathbf{S}-\mathbf{I}\right): \boldsymbol{\sigma}^{\prime}}=-|\Omega| \mathbf{E}:\left(2 \mathbf{C}_{0}-\mathbf{C}_{0}: \overline{\mathbf{S}}: \mathbf{C}_{0}-\mathbf{C}_{\mathrm{MBC}}^{\mathrm{app}}\left(\mathbf{C}_{0}\right)\right): \mathbf{E},
$$

and, using (36)

$$
\begin{aligned}
\mathcal{W}^{*}(\boldsymbol{\sigma}) & =\frac{1}{2}|\Omega| \mathbf{E}: \overline{\left(\mathbf{C}_{0}: \mathbf{S}-\mathbf{I}\right): \boldsymbol{\sigma}^{\prime}}+\frac{1}{2}|\Omega| \mathbf{E}:\left(\mathbf{C}_{0}: \overline{\mathbf{S}}: \mathbf{C}_{0}-\mathbf{C}_{0}\right): \mathbf{E} \\
& =\frac{1}{2}|\Omega| \mathbf{E}:\left(\mathbf{C}_{0}-\mathbf{C}_{\mathrm{MBC}}^{\mathrm{app}}\left(\mathbf{C}_{0}\right)\right): \mathbf{E} .
\end{aligned}
$$

In other words

$$
\min _{\boldsymbol{\sigma} \in \mathbf{C}_{0}: \mathbf{E}+\mathbb{K}^{+}} \mathcal{W}^{*}(\boldsymbol{\sigma})=-\frac{1}{2}|\Omega| \mathbf{E}:\left(\mathbf{C}_{\mathrm{MBC}}^{\mathrm{app}}\left(\mathbf{C}_{0}\right)-\mathbf{C}_{0}\right): \mathbf{E} .
$$

\subsection{Applications: properties of the apparent stiffness}

In the present section, we use the previous results to prove some properties of the apparent stiffness $\mathbf{C}_{\mathrm{MBC}}^{\mathrm{app}}\left(\mathbf{C}_{0}\right)$ defined by Eq. (11). These properties have already been stated without proof in Sec. 2. 
Lower-bound on the apparent stiffness. It can readily be shown that $\mathbf{C}_{\mathrm{SUBC}}^{\mathrm{app}} \leq \mathbf{C}_{\mathrm{MBC}}^{\mathrm{app}}\left(\mathbf{C}_{0}\right)$. To this end, we define on $\mathbb{R}^{d}$ a trial stress $\sigma_{\text {trial }}$, statically admissible with $\mathbf{E}$ as follows

1. In $\Omega, \sigma_{\text {trial }}=\sigma_{\mathrm{SUBC}}$, where $\sigma_{\mathrm{SUBC}}$ is the stress arising from Problem SUBC [see Eqs. (1) and (2)], and $\boldsymbol{\Sigma}$ is chosen so as to ensure that the average strain in $\Omega$ is $\mathbf{E}$.

2. Outside $\Omega, \sigma_{\text {trial }}=\mathbf{C}_{0}: \mathbf{E}$.

It is readily verified that $\sigma_{\text {trial }} \in \mathbf{C}_{0}: \mathbf{E}+\mathbb{S}^{\dagger}$. Besides, for $\sigma=\sigma_{\text {trial }}$, the integrand in Eq. (10) vanishes in $\mathbb{R}^{d} \backslash \Omega$, and a straightforward application of Hill's lemma in the bounded domain $\Omega$ leads to

$$
\mathcal{W}^{*}\left(\sigma_{\text {trial }}\right)=\frac{1}{2}|\Omega| \mathbf{E}:\left(\mathbf{C}_{0}-\mathbf{C}_{\mathrm{SUBC}}^{\mathrm{app}}\right): \mathbf{E} .
$$

From Theorem $9, \mathcal{W}^{*}\left(\sigma_{\mathrm{MBC}}\right) \leq \mathcal{W}^{*}\left(\sigma_{\text {trial }}\right)$, where $\sigma_{\mathrm{MBC}}$ is the stress arising from Problem MBC. The proposed lower-bound then results from the comparison of Eqs. (38) and (39).

Upper-bound on the apparent stiffness. It can readily be shown that $\mathbf{C}_{\mathrm{MBC}}^{\mathrm{app}}\left(\mathbf{C}_{0}\right) \leq \mathbf{C}_{\mathrm{KUBC}}^{\mathrm{app}}$. To this end, we define on $\mathbb{R}^{d}$ a trial strain $\boldsymbol{\varepsilon}_{\text {trial }}$, kinematically admissible with $\mathbf{E}$ as follows

1. In $\Omega, \varepsilon_{\text {trial }}=\varepsilon_{\mathrm{KUBC}}$, where $\boldsymbol{\varepsilon}_{\mathrm{KUBC}}$ is the displacement arising from Problem KUBC [see Eqs. (1) and (3)].

2. Outside $\Omega, \boldsymbol{\varepsilon}_{\text {trial }}=\mathbf{E}$.

Is is readily verified that $\boldsymbol{\varepsilon}_{\text {trial }} \in \mathbf{E}+\mathbb{K}^{\dagger}$. Besides, for $\boldsymbol{\varepsilon}=\boldsymbol{\varepsilon}_{\text {trial }}$, the integrand in Eq. (9) vanishes in $\mathbb{R}^{d} \backslash \Omega$, and a straightforward application of Hill's lemma in the bounded domain $\Omega$ leads to

$$
\mathcal{W}\left(\boldsymbol{\varepsilon}_{\text {trial }}\right)=\frac{1}{2}|\Omega| \mathbf{E}:\left(\mathbf{C}_{\mathrm{KUBC}}^{\mathrm{app}}-\mathbf{C}_{0}\right): \mathbf{E} .
$$

From Theorem $8, \mathcal{W}\left(\varepsilon_{\mathrm{MBC}}\right) \leq \mathcal{W}\left(\varepsilon_{\text {trial }}\right)$, where $\varepsilon_{\mathrm{MBC}}$ is the strain arising from Problem MBC. The proposed upper-bound then results from the comparison of Eqs. (34) and (40).

Extreme values of $\mathbf{C}_{0}$. In the present paragraph, we address the two extreme cases of a reference medium with vanishing stiffness and a rigid reference medium. Intuitively, it is clear that boundary condition (7) wins over the boundary condition at infinity (6d) for a soft reference medium. In other words, Problem SUBC is retrieved in that case. Conversely, for a rigid reference medium, the boundary condition at infinity (6d) wins over boundary condition (7), and Problem KUBC is retrieved. This is expressed more formally in the following two theorems.

Theorem 10. For any macroscopic strain $\mathbf{E}$, there exists $\gamma_{\mathrm{SUBC}}>0$, which does not depend on the stiffness $\mathbf{C}_{0}$ of the reference medium, such that

$$
0 \leq \mathbf{E}:\left(\mathbf{C}_{\mathrm{MBC}}^{\mathrm{app}}\left(\mathbf{C}_{0}\right)-\mathbf{C}_{\mathrm{SUBC}}^{\mathrm{app}}\right): \mathbf{E} \leq \gamma_{\mathrm{SUBC}} \beta_{0},
$$

where $\beta_{0}$ is the largest eigenvalue of $\mathbf{C}_{0}$.

Theorem 11. For any macroscopic strain $\mathbf{E}$, there exists $\gamma_{\mathrm{KUBC}}>0$, which does not depend on the stiffness $\mathbf{C}_{0}$ of the reference medium, such that

$$
0 \leq \mathbf{E}:\left(\mathbf{C}_{\mathrm{KUBC}}^{\mathrm{app}}-\mathbf{C}_{\mathrm{MBC}}^{\mathrm{app}}\left(\mathbf{C}_{0}\right)\right): \mathbf{E} \leq \frac{\gamma_{\mathrm{KUBC}}}{\alpha_{0}},
$$

where $\alpha_{0}$ is the smallest eigenvalue of $\mathbf{C}_{0}$.

It should be noted that in both cases, the positivity of the quantities under consideration results from the lowerand upper-bounds proposed above. The proof of Theorems 10 and 11 can be found in Appendix D. Eq. (12) is then a direct consequence of these theorems.

In Sec. 6 below, we show that the principle of Hashin and Shtrikman (1962a) can be extended to the modified Lippmann-Schwinger equation (8) introduced in Sec. 3.4 for ellipsoidal SVEs. 


\section{Principle of Hashin and Shtrikman associated with the modified Lippmann-Schwinger equation}

In the present section, it is assumed that the SVE $\Omega$ is ellipsoidal; besides, $\tau$ denotes the solution to the modified Lippmann-Schwinger equation (8). For $\varpi \in \mathbb{L}^{2}(\Omega)$, the functional $\mathcal{H}_{\mathrm{MBC}}$ of Hashin and Shtrikman is defined by Eq. (13), and it is readily verified [using Eq. (8)] that the gradient of $\mathcal{H}_{\mathrm{MBC}}$ is null at $\boldsymbol{\tau}$. In other words, $\boldsymbol{\tau}$ is a critical point of the functional $\mathcal{H}_{\mathrm{MBC}}$; furthermore, the value of $\mathcal{H}_{\mathrm{MBC}}$ at $\tau$ is known. Indeed, from Eq. (8), $\mathcal{H}_{\mathrm{MBC}}(\tau)=\frac{1}{2} \bar{\tau}: \mathbf{E}$ and, from $\sigma=\mathbf{C}_{0}: \varepsilon+\tau$ and Eq. (11)

$$
\bar{\tau}=\left(\mathbf{C}_{\mathrm{MBC}}^{\mathrm{app}}\left(\mathbf{C}_{0}\right)-\mathbf{C}_{0}\right): \mathbf{E},
$$

and we finally find

$$
\mathcal{H}_{\mathrm{MBC}}(\boldsymbol{\tau})=\frac{1}{2} \mathbf{E}:\left(\mathbf{C}_{\mathrm{MBC}}^{\mathrm{app}}\left(\mathbf{C}_{0}\right)-\mathbf{C}_{0}\right): \mathbf{E} .
$$

In order to characterize the nature (minimum, maximum or saddle point) of this stationary point, further assumptions must be made on the local stiffness. More precisely

Theorem 12 (Principle of Hashin and Shtrikman). If the reference medium is stiffer (resp. softer) than the heterogeneous material, then $\mathcal{H}_{\mathrm{MBC}}$ is minimum (resp. maximum) at $\tau$. In other words

1. if $\mathbf{C} \leq \mathbf{C}_{0}$ in $\Omega$, then $\tau$ is the unique minimizer of $\mathcal{H}_{\mathrm{MBC}}$, and for all $\varpi \in \mathbb{L}^{2}(\Omega)$

$$
\frac{1}{2} \mathbf{E}: \mathbf{C}_{\mathrm{MBC}}^{\mathrm{app}}\left(\mathbf{C}_{0}\right): \mathbf{E} \leq \frac{1}{2} \mathbf{E}: \mathbf{C}_{0}: \mathbf{E}+\mathcal{H}_{\mathrm{MBC}}(\varpi),
$$

2. if $\mathbf{C} \geq \mathbf{C}_{0}$ in $\Omega$, then $\boldsymbol{\tau}$ is the unique maximizer of $\mathcal{H}_{\mathrm{MBC}}$, and for all $\boldsymbol{\varpi} \in \mathbb{L}^{2}(\Omega)$

$$
\frac{1}{2} \mathbf{E}: \mathbf{C}_{\mathrm{MBC}}^{\mathrm{app}}\left(\mathbf{C}_{0}\right): \mathbf{E} \geq \frac{1}{2} \mathbf{E}: \mathbf{C}_{0}: \mathbf{E}+\mathcal{H}_{\mathrm{MBC}}(\varpi),
$$

Proof of Theorem 12. We introduce the bilinear form $a$, defined as follows for $\varpi_{1}, \varpi_{2} \in \mathbb{L}^{2}(\Omega)$

$$
a\left(\varpi_{1}, \varpi_{2}\right)=\overline{\varpi_{1}:\left(\mathbf{C}-\mathbf{C}_{0}\right)^{-1}: \varpi_{2}}+\overline{\varpi_{1}:\left(\boldsymbol{\Gamma}_{0}^{\infty} *\left(\varpi_{2}-\chi \overline{\boldsymbol{\varpi}}_{2}\right)\right)},
$$

so that $\mathcal{H}_{\mathrm{MBC}}(\boldsymbol{\varpi})=\mathbf{E}: \overline{\boldsymbol{\varpi}}-\frac{1}{2} a(\boldsymbol{\varpi}, \boldsymbol{\varpi})$. In Appendix E, two alternative expressions of the bilinear form $a$ are proposed [see Lemma 2, Eq. (E.2) and Lemma 3, Eq. (E.4)], which readily lead to the following inequalities

$$
\overline{\varpi:\left(\mathbf{C}-\mathbf{C}_{0}\right)^{-1}: \varpi} \leq a(\varpi, \varpi) \leq \overline{\varpi: \mathbf{S}_{0}:\left(\mathbf{S}_{0}-\mathbf{S}\right)^{-1}: \mathbf{S}_{0}: \varpi},
$$

for all $\varpi \in \mathbb{L}^{2}(\Omega)$. Then, in case 1 (resp. 2), $-a(\varpi, \varpi)$ is a positive (resp. negative) quadratic form. Therefore, its unique critical point is a minimum (resp. maximum).

At this point, it should be emphasized again that, despite the seemingly similar expressions, our approach differs significantly from the approach of Willis (1977). Indeed, in Ref. (Willis, 1977), $\mathcal{H}_{\mathrm{MBC}}$ [see Eq. (13)] is used as an approximation of the functional of Hashin and Shtrikman $\mathcal{H}_{\mathrm{KUBC}}$ associated with Problem KUBC [see Eq. (18)]. This approximation is deemed valid in the limit $|\Omega| \rightarrow+\infty$. In our approach, $\mathcal{H}_{\mathrm{MBC}}$ is the Hashin and Shtrikman functional associated with Problem MBC, and Theorem 12 provides rigorous bounds on $\mathbf{C}_{\mathrm{MBC}}^{\text {app }}\left(\mathbf{C}_{0}\right)$ (not $\mathbf{C}_{\mathrm{KUBC}}^{\text {app }}$ ), regardless of the size of the SVE $\Omega$.

Since $\mathbf{C}_{\mathrm{MBC}}^{\mathrm{app}}\left(\mathbf{C}_{0}\right) \rightarrow \mathbf{C}^{\mathrm{eff}}$ as $|\Omega| \rightarrow+\infty$ (see Sec. 2), the bounds that are derived from the application of Theorem 12 can be regarded as bounds on the effective stiffness in the limit $|\Omega| \rightarrow+\infty$.

The results presented in this section have deep implications for the numerical approximation of Eq. (8). Indeed, provided that the assumptions of Theorem 12 are verified, any approximation $\varpi$ of the true polarization $\tau$ provides a bound on the apparent elastic energy [see Eqs. (41) and (42)]. Since $\mathcal{H}_{\mathrm{MBC}}$ involves the Green operator of the unbounded domain $\Gamma_{0}^{\infty}$ only [see Eq. (13)], numerical evaluation of $\mathcal{H}_{\mathrm{MBC}}(\varpi)$ is rather simple, and optimizations can be proposed which account for the fact that $\boldsymbol{\Gamma}_{0}^{\infty}$ is translation-invariant. 


\section{Conclusion}

A new auxiliary problem for the determination of the effective elastic properties of random heterogeneous materials has been introduced. It is obtained as follows. The SVE is embedded in an infinite, homogeneous reference medium, submitted to a uniform strain at infinity. Meanwhile, tractions are applied to the boundary of the SVE [see Eq. (7)], ensuring that the imposed strain at infinity coincides with the average strain over the SVE.

We have shown that under mild assumptions, this new auxiliary problem is well-posed. This required the introduction of a suitable mathematical framework for the analysis of elasticity problems in unbounded domains. For ellipsoidal SVEs, the new auxiliary problem is equivalent to a modified Lippmann-Schwinger equation which involves the Green operator for strains of the infinite (rather than bounded) body [see Eq. (8)]. In turn, this integral equation can be associated with an energy principle of the Hashin and Shtrikman (1962a) type, allowing for the computation of rigorous bounds on the apparent stiffness.

The apparent stiffness defined through this new auxiliary problem is symmetric, positive definite. It is asymptotically consistent, in the sense that the effective stiffness is retrieved in the limit of large SVEs. This result provides a rigorous justification to the approximation of the Green operator introduced by Willis (1977).

For SVEs of finite size, the value of the apparent stiffness depends on the stiffness of the reference medium. For infinitely soft reference media, it coincides with the apparent stiffness defined through static uniform boundary conditions. Conversely, for infinitely stiff reference media, it coincides with the apparent stiffness defined through static uniform boundary conditions. In this sense, it "interpolates" between these two extreme values.

The results presented in this paper raise a number of questions, which will be investigated in future work. First, the convergence rate (with respect to the size of the SVE) of the apparent stiffness to the effective stiffness should be estimated. Second, the apparent stiffness defined through the new auxiliary problem depends on the stiffness of the reference medium. How to select the "best" (in a sense to be made more precise) reference medium remains an open question. Third, this apparent stiffness depends on the shape of the SVE, although this dependence vanishes in the limit of large SVEs. In the case of matrix-inclusions composites, this dependence should be assessed in conjunction with the shape of the inclusions.

Finally, it should be noticed that the modified Lippmann-Schwinger equation introduced in the present paper can readily be discretized through Galerkin techniques. The resulting numerical scheme is close to the standard Equivalent Inclusion Method proposed by Moschovidis and Mura (1975), with the additional possibility of computing rigorous bounds on the apparent stiffness (provided the reference medium satisfies the assumptions of the principle of Hashin and Shtrikman, see Theorem 12). Such a numerical scheme, based on piecewise polynomial polarizations, will be presented in a separate publication.

\section{References}

Amrouche, C., Ciarlet, P.G., Gratie, L., Kesavan, S., 2006. On the characterizations of matrix fields as linearized strain tensor fields. Journal de Mathématiques Pures et Appliquées 86, 116-132.

Amrouche, C., Dambrine, M., Raudin, Y., 2012. An theory of linear elasticity in the half-space. Journal of Differential Equations $253,906-932$.

Benveniste, Y., 1987. A new approach to the application of Mori-Tanaka's theory in composite materials. Mechanics of Materials 6, 147-157.

Brisard, S., Dormieux, L., 2010. FFT-based methods for the mechanics of composites: A general variational framework. Computational Materials Science 49, 663-671.

Brisard, S., Dormieux, L., 2012. Combining Galerkin approximation techniques with the principle of Hashin and Shtrikman to derive a new FFT-based numerical method for the homogenization of composites. Computer Methods in Applied Mechanics and Engineering 217-220, 197-212.

Buryachenko, V.A., 2007. Micromechanics of Heterogeneous Materials. Springer.

Christensen, R.M., Lo, K.H., 1979. Solutions for effective shear properties in three phase sphere and cylinder models. Journal of the Mechanics and Physics of Solids 27, 315-330.

Eshelby, J.D., 1957. The determination of the elastic field of an ellipsoidal inclusion, and related problems. Proceedings of the Royal Society of London. Series A, Mathematical and Physical Sciences 241, 376-396.

Fond, C., Géhant, S., Schirrer, R., 2002. Effects of mechanical interactions on the hydrostatic stress in randomly distributed rubber particles in an amorphous polymer matrix. Polymer 43, 909-919.

Fond, C., Riccardi, A., Schirrer, R., Montheillet, F., 2001. Mechanical interaction between spherical inhomogeneities: an assessment of a method based on the equivalent inclusion. European Journal of Mechanics - A/Solids 20, 59-75.

Gusev, A.A., 1997. Representative volume element size for elastic composites: A numerical study. Journal of the Mechanics and Physics of Solids 45, 1449-1459.

Hashin, Z., Shtrikman, S., 1962a. On some variational principles in anisotropic and nonhomogeneous elasticity. Journal of the Mechanics and Physics of Solids 10, 335-342. 
Hashin, Z., Shtrikman, S., 1962b. A variational approach to the theory of the elastic behaviour of polycrystals. Journal of the Mechanics and Physics of Solids 10, 343-352.

Hervé, E., Zaoui, A., 1993. n-layered inclusion-based micromechanical modelling. International Journal of Engineering Science 31, 1-10.

Hill, R., 1963. Elastic properties of reinforced solids: Some theoretical principles. Journal of the Mechanics and Physics of Solids 11, $357-372$.

Hill, R., 1967. The essential structure of constitutive laws for metal composites and polycrystals. Journal of the Mechanics and Physics of Solids 15 , 79-95.

Kanaun, S.K., Levin, V.M., 2008. Self-Consistent Methods for Composites Vol.1: Static Problems. volume 148 of Solid Mechanics and its Applications. Springer.

Kondrat'ev, V.A., Oleinik, O.A., 1988. Boundary-value problems for the system of elasticity theory in unbounded domains. korn's inequalities. Russian Mathematical Surveys 43, 65.

Kröner, E., 1977. Bounds for effective elastic moduli of disordered materials. Journal of the Mechanics and Physics of Solids 25, 137-155.

Mandel, J., 1972. Plasticité classique et viscoplasticité. Number 97 in CISM courses and lectures, Springer, Berlin.

McCartney, L.N., 2010. Maxwell's far-field methodology predicting elastic properties of multiphase composites reinforced with aligned transversely isotropic spheroids. Philosophical Magazine 90, 4175-4207.

McCartney, L.N., Kelly, A., 2008. Maxwell's far-field methodology applied to the prediction of properties of multi-phase isotropic particulate composites. Proceedings: Mathematical, Physical and Engineering Sciences 464, 423-446.

Michel, J.C., Moulinec, H., Suquet, P., 2001. A computational scheme for linear and non-linear composites with arbitrary phase contrast. International Journal for Numerical Methods in Engineering 52, 139-160.

Milton, G.W., 2002. The Theory of Composites. Cambridge Monographs on Applied and Computational Mathematics, Cambridge University Press, The Edinburgh Building, Cambridge CB2 8RU, UK.

Moreau, J.J., 1979. Duality characterization of strain tensor distributions in an arbitrary open set. Journal of Mathematical Analysis and Applications $72,760-770$.

Mori, T., Tanaka, K., 1973. Average stress in matrix and average elastic energy of materials with misfitting inclusions. Acta Metallurgica 21, 571-574.

Moschovidis, Z.A., Mura, T., 1975. Two-ellipsoidal inhomogeneities by the equivalent inclusion method. Journal of Applied Mechanics 42, 847-852.

Moulinec, H., Suquet, P., 1994. A fast numerical method for computing the linear and nonlinear properties of composites. Comptes-rendus de l'Académie des sciences série II 318, 1417-1423.

Moulinec, H., Suquet, P., 1998. A numerical method for computing the overall response of nonlinear composites with complex microstructure. Computer Methods in Applied Mechanics and Engineering 157, 69-94.

Ostoja-Starzewski, M., 2006. Material spatial randomness: From statistical to representative volume element. Probabilistic Engineering Mechanics 21, 112-132.

Ponte Castañeda, P., Willis, J.R., 1995. The effect of spatial distribution on the effective behavior of composite materials and cracked media. Journal of the Mechanics and Physics of Solids 43, 1919-1951.

Rodin, G.J., Hwang, Y.L., 1991. On the problem of linear elasticity for an infinite region containing a finite number of non-intersecting spherical inhomogeneities. International Journal of Solids and Structures 27, 145-159.

Sab, K., 1992. On the homogenization and the simulation of random materials. European Journal of Mechanics - A/Solids 11, 585-607.

Suquet, P. (Ed.), 1997. Continuum Micromechanics. Number 377 in CISM courses and lectures, Springer Wien New-York.

Torquato, S., 1997. Effective stiffness tensor of composite media-I. Exact series expansions. Journal of the Mechanics and Physics of Solids 45, $1421-1448$.

Torquato, S., 2002. Random heterogeneous materials: microstructure and macroscopic properties. Springer-Verlag.

Walpole, L.J., 1969. On the overall elastic moduli of composite materials. Journal of the Mechanics and Physics of Solids 17, 235-251.

Willis, J.R., 1977. Bounds and self-consistent estimates for the overall properties of anisotropic composites. Journal of the Mechanics and Physics of Solids 25, 185-202.

Zeller, R., Dederichs, P.H., 1973. Elastic constants of polycrystals. Physica Status Solidi (B) 55, 831-842.

\section{Appendix A. On the infinite body Green's operator for strains}

For a sufficiently regular prestress $\varpi$, the Green's operator for strains $\boldsymbol{\Gamma}_{0}^{\infty}$ associated with the isotropic reference medium $\mathbf{C}_{0}$ is classically decomposed into a singular part $\mathbf{P}_{0}$ and a regular part $\mathbf{Q}_{0}$

$$
\left(\Gamma_{0}^{\infty} * \varpi\right)(\mathbf{x})=\mathbf{P}_{0}: \varpi(\mathbf{x})+\lim _{\delta \rightarrow 0} \int_{\substack{\mathbf{y} \in \mathbb{R}^{d} \\\|\mathbf{y}-\mathbf{x}\| \geq \delta}} \mathbf{Q}_{0}(\mathbf{x}-\mathbf{y}): \varpi(\mathbf{y}) d \mathbf{y} .
$$

Literal expressions of $\mathbf{P}_{0}$ and $\mathbf{Q}_{0}(\mathbf{r})$ can be found in reference textbooks for isotropic reference media (see e.g. Torquato, 2002; Buryachenko, 2007; Kanaun and Levin, 2008). For the sake of completeness, they are recalled below, for $d=2$ (plane strain elasticity), and $d=3$ (three-dimensional elasticity). $\mathbf{P}_{0}$ is the Hill tensor of the $d$-dimensional sphere

$$
\begin{array}{ll}
d=2: & \mathbf{P}_{0}=\frac{1-2 v_{0}}{4 \mu_{0}\left(1-v_{0}\right)} \mathbf{J}+\frac{3-4 v_{0}}{8 \mu_{0}\left(1-v_{0}\right)} \mathbf{K}, \\
d=3: & \mathbf{P}_{0}=\frac{1-2 v_{0}}{6 \mu_{0}\left(1-v_{0}\right)} \mathbf{J}+\frac{4-5 v_{0}}{15 \mu_{0}\left(1-v_{0}\right)} \mathbf{K},
\end{array}
$$


where $\mu_{0}$ (resp. $v_{0}$ ) is the shear modulus (resp. Poisson ratio) of the reference medium; $\mathbf{J}=\frac{1}{d} \mathbf{i} \otimes \mathbf{i}(\operatorname{resp} . \mathbf{K}=\mathbf{I}-\mathbf{J})$ is the classical spherical (resp. deviatoric) fourth-rank projection tensor (i: second-rank identity tensor; I: fourth-rank identity tensor). For $d=2$, the regular part of $\boldsymbol{\Gamma}_{0}^{\infty}$ reads

$$
\begin{aligned}
\mathbf{Q}_{0}(\mathbf{r})= & \frac{1}{8 \pi \mu_{0}\left(1-v_{0}\right) r^{2}}\left[-\delta_{i j} \delta_{k l}+\left(1-2 v_{0}\right)\left(\delta_{i k} \delta_{j l}+\delta_{i l} \delta_{j k}\right)+2\left(\delta_{i j} n_{k} n_{l}+\delta_{k l} n_{i} n_{j}\right)\right. \\
& \left.+2 v_{0}\left(\delta_{i k} n_{j} n_{l}+\delta_{i l} n_{j} n_{k}+\delta_{j k} n_{i} n_{l}+\delta_{j l} n_{i} n_{k}\right)-8 n_{i} n_{j} n_{k} n_{l}\right] \mathbf{e}_{i} \otimes \mathbf{e}_{j} \otimes \mathbf{e}_{k} \otimes \mathbf{e}_{l},
\end{aligned}
$$

and for $d=3$

$$
\begin{aligned}
\mathbf{Q}_{0}(\mathbf{r})= & \frac{1}{16 \pi \mu_{0}\left(1-v_{0}\right) r^{3}}\left[-\delta_{i j} \delta_{k l}+\left(1-2 v_{0}\right)\left(\delta_{i k} \delta_{j l}+\delta_{i l} \delta_{j k}\right)+3\left(\delta_{i j} n_{k} n_{l}+\delta_{k l} n_{i} n_{j}\right)\right. \\
& \left.+3 v_{0}\left(\delta_{i k} n_{j} n_{l}+\delta_{i l} n_{j} n_{k}+\delta_{j k} n_{i} n_{l}+\delta_{j l} n_{i} n_{k}\right)-15 n_{i} n_{j} n_{k} n_{l}\right] \mathbf{e}_{i} \otimes \mathbf{e}_{j} \otimes \mathbf{e}_{k} \otimes \mathbf{e}_{l}
\end{aligned}
$$

where $r=\|\mathbf{r}\|$ and $\mathbf{n}=\mathbf{r} / r$.

\section{Appendix B. On the generalized theorem of Eshelby}

Lemma 1. Let $\varpi_{1}, \varpi_{2} \in \mathbb{L}^{2}\left(\mathbb{R}^{d}\right)$; then $\left\langle\varpi_{1}, \Gamma_{0}^{\infty} * \varpi_{2}\right\rangle=\left\langle\varpi_{2}, \Gamma_{0}^{\infty} * \varpi_{1}\right\rangle$.

Proof of Lemma 1. For $i=1,2$, let $\varepsilon_{i}=-\Gamma_{0}^{\infty} * \varpi_{i} \in \mathbb{K}$ and $\sigma_{i}=\mathbf{C}_{0}: \boldsymbol{\varepsilon}_{i}+\varpi_{i} \in \mathbb{S}$. Then, using Theorem 4 with $\sigma_{1}$ and $\boldsymbol{\varepsilon}_{2}$

$$
\left\langle\varpi_{1}, \Gamma_{0}^{\infty} * \varpi_{2}\right\rangle=\left\langle\mathbf{C}_{0}: \varepsilon_{1}-\sigma_{1}, \boldsymbol{\varepsilon}_{2}\right\rangle=\left\langle\mathbf{C}_{0}: \varepsilon_{1}, \boldsymbol{\varepsilon}_{2}\right\rangle,
$$

which completes the proof, since the expression in the right-hand side is symmetric in $\boldsymbol{\varepsilon}_{1}$ and $\boldsymbol{\varepsilon}_{2}$.

Proof of Theorem 2. Let $\varpi^{\prime}$ be a constant, symmetric tensor. Then, using Lemma 1

$$
\varpi^{\prime}: \overline{\Gamma_{0}^{\infty} * \varpi}=\overline{\left(\chi \varpi^{\prime}\right):\left(\boldsymbol{\Gamma}_{0}^{\infty} * \varpi\right)}=|\Omega|^{-1}\left\langle\chi \varpi^{\prime}, \Gamma_{0}^{\infty} * \varpi\right\rangle=|\Omega|^{-1}\left\langle\varpi, \Gamma_{0}^{\infty} *\left(\chi \varpi^{\prime}\right)\right\rangle .
$$

Observing that $\varpi$ is supported in $\Omega$, it is found that the last scalar product reduces to a volume average over $\Omega$. Then, from Theorem 1 ( $\Omega$ being ellipsoidal)

$$
\varpi^{\prime}: \overline{\Gamma_{0}^{\infty} * \varpi}=\overline{\varpi:\left(\Gamma_{0}^{\infty} *\left(\chi \varpi^{\prime}\right)\right)}=\overline{\varpi: \mathbf{P}_{\Omega}: \varpi^{\prime}}=\varpi^{\prime}: \mathbf{P}_{\Omega}: \bar{\varpi},
$$

which completes the proof, since the above identity holds for all $\varpi^{\prime}$.

\section{Appendix C. On the minimum energy principles}

Proof of Theorem 8. We first consider a solution $\boldsymbol{\varepsilon} \in \mathbf{E}+\mathbb{K}^{\dagger}$ of Problem MBC, and show that the potential energy $\mathcal{W}$ is minimum at $\boldsymbol{\varepsilon}$. To do so, we pick $\delta \boldsymbol{\varepsilon} \in \mathbb{K}^{\dagger}$ and show that $\mathcal{W}(\boldsymbol{\varepsilon}) \leq \mathcal{W}(\boldsymbol{\varepsilon}+\delta \boldsymbol{\varepsilon})$. From Eq. (9)

$$
\mathcal{W}(\boldsymbol{\varepsilon}+\delta \boldsymbol{\varepsilon})=\mathcal{W}(\boldsymbol{\varepsilon})+\frac{1}{2}\langle\delta \boldsymbol{\varepsilon}, \mathbf{C}: \delta \boldsymbol{\varepsilon}\rangle+\left\langle\boldsymbol{\sigma}-\mathbf{C}_{0}: \mathbf{E}, \delta \boldsymbol{\varepsilon}\right\rangle,
$$

and the last term vanishes from Theorem 5 since $\sigma-\mathbf{C}_{0}: \mathbf{E} \in \mathbb{S}^{\dagger}$ and $\delta \boldsymbol{\varepsilon} \in \mathbb{K}^{\dagger}$. Therefore,

$$
\mathcal{W}(\boldsymbol{\varepsilon}+\delta \boldsymbol{\varepsilon})=\mathcal{W}(\boldsymbol{\varepsilon})+\frac{1}{2}\langle\delta \boldsymbol{\varepsilon}, \mathbf{C}: \delta \boldsymbol{\varepsilon}\rangle,
$$

which proves that $\mathcal{W}$ is minimum at $\boldsymbol{\varepsilon}$, since the second term is positive.

Conversely, we now consider $\boldsymbol{\varepsilon} \in \mathbf{E}+\mathbb{K}^{\dagger}$ which minimizes $\mathcal{W}$ over $\mathbf{E}+\mathbb{K}^{\dagger}$, and show that $\boldsymbol{\varepsilon}$ solves Problem MBC. We define $\boldsymbol{\sigma}=\mathbf{C}: \boldsymbol{\varepsilon}$; since $\boldsymbol{\varepsilon}$ is a solution to (33), for all $\delta \boldsymbol{\varepsilon} \in \mathbb{K}^{\dagger}$,

$$
\begin{aligned}
0 & =\langle\boldsymbol{\sigma}-\mathbf{C}: \mathbf{E}, \delta \boldsymbol{\varepsilon}\rangle+|\Omega| \mathbf{E}: \overline{\left(\mathbf{C}-\mathbf{C}_{0}\right): \delta \boldsymbol{\varepsilon}}=\langle\boldsymbol{\sigma}-\mathbf{C}: \mathbf{E}, \delta \boldsymbol{\varepsilon}\rangle+\left\langle\left(\mathbf{C}-\mathbf{C}_{0}\right): \mathbf{E}, \delta \boldsymbol{\varepsilon}\right\rangle \\
& =\left\langle\sigma-\mathbf{C}_{0}: \mathbf{E}, \delta \boldsymbol{\varepsilon}\right\rangle,
\end{aligned}
$$

in other words, $\sigma-\mathbf{C}_{0}: \mathbf{E} \in\left(\mathbb{K}^{\dagger}\right)^{\perp}$. Theorem 5 then shows that $\boldsymbol{\sigma}$ is statically admissible with $\mathbf{E}$, and that $\boldsymbol{\varepsilon}$ solves Problem MBC. 
Proof of Theorem 9. We first consider a solution $\varepsilon \in \mathbf{E}+\mathbb{K}^{\dagger}$ of Problem MBC, and show that the complementary energy $\mathcal{W}^{*}$ is minimum at $\boldsymbol{\sigma}=\mathbf{C}: \boldsymbol{\varepsilon} \in \mathbf{C}_{0}: \mathbf{E}+\mathbb{K}^{\dagger}$. To do so, we pick $\delta \boldsymbol{\sigma} \in \mathbb{S}^{\dagger}$ and show that $\mathcal{W}^{*}(\boldsymbol{\sigma}) \leq \mathcal{W}^{*}(\boldsymbol{\sigma}+\delta \boldsymbol{\sigma})$. From Eq. (10)

$$
\mathcal{W}^{*}(\sigma+\delta \sigma)=\mathcal{W}^{*}(\sigma)+\frac{1}{2}\langle\delta \sigma, \mathbf{S}: \delta \boldsymbol{\sigma}\rangle+\langle\boldsymbol{\varepsilon}-\mathbf{E}, \delta \sigma\rangle,
$$

and the last term vanishes from Theorem 5 since $\varepsilon-\mathbf{E} \in \mathbb{K}^{\dagger}$ and $\delta \sigma \in \mathbb{S}^{\dagger}$. Therefore,

$$
\mathcal{W}^{*}(\sigma+\delta \sigma)=\mathcal{W}^{*}(\sigma)+\frac{1}{2}\langle\delta \sigma, \mathbf{S}: \delta \sigma\rangle,
$$

which proves that $W^{*}$ is minimum at $\sigma$, since the second term is positive.

Conversely, we now consider $\sigma \in \mathbf{C}_{0}: \mathbf{E}+\mathbb{S}^{\dagger}$ which minimizes $\mathcal{W}^{*}$ over $\mathbf{C}_{0}: \mathbf{E}+\mathbb{S}^{\dagger}$, and show that $\boldsymbol{\varepsilon}=\mathbf{S}: \sigma$ solves Problem MBC; since $\sigma$ is a solution to (37), for all $\delta \sigma \in \mathbb{S}^{\dagger}$,

$$
\begin{aligned}
0 & =\left\langle\boldsymbol{\varepsilon}-\mathbf{S}: \mathbf{C}_{0}: \mathbf{E}, \delta \boldsymbol{\sigma}\right\rangle+|\Omega| \mathbf{E}: \overline{\left(\mathbf{C}_{0}: \mathbf{S}-\mathbf{I}\right): \delta \boldsymbol{\varepsilon}} \\
& =\left\langle\boldsymbol{\varepsilon}-\mathbf{S}: \mathbf{C}_{0}: \mathbf{E}, \delta \boldsymbol{\sigma}\right\rangle+\left\langle\left(\mathbf{S}: \mathbf{C}_{0}-\mathbf{I}\right): \mathbf{E}, \delta \boldsymbol{\varepsilon}\right\rangle=\langle\boldsymbol{\varepsilon}-\mathbf{E}, \delta \boldsymbol{\sigma}\rangle,
\end{aligned}
$$

in other words, $\boldsymbol{\varepsilon}-\mathbf{E} \in\left(\mathbb{S}^{\dagger}\right)^{\perp}$. Theorem 5 then shows that $\boldsymbol{\varepsilon}$ is kinematically admissible with $\mathbf{E}$, and that $\boldsymbol{\varepsilon}$ solves Problem MBC.

\section{Appendix D. On the apparent stiffness when the stiffness of the reference medium takes extreme values}

In the present section, it will prove convenient to (strictly) include the bounded domain $\Omega$ in a larger domain $\Omega$. Furthermore, the positive definite stiffness $\mathbf{C}_{\text {aux }}$ of an auxiliary material is introduced; $\mathbf{C}_{\mathrm{aux}}$ is fixed (independent of $\mathbf{C}_{0}$ ).

Proof of Theorem 10. In order to invoke the principle of minimum potential energy (see Theorem 8), we define on $\mathbb{R}^{d}$ a kinematically admissible trial strain $\boldsymbol{\varepsilon}_{\text {trial }}$ as follows:

1. In $\Omega, \varepsilon_{\text {trial }}=\varepsilon_{\text {SUBC }}$, where $\varepsilon_{\text {SUBC }}$ is the strain arising from Problem SUBC [see Eqs. (1) and (2)], and $\boldsymbol{\Sigma}$ is chosen so as to ensure that $\overline{\varepsilon_{\mathrm{SUBC}}}=\mathbf{E}$.

2. In $\tilde{\Omega} \backslash \Omega, \boldsymbol{\varepsilon}_{\text {trial }}=\mathbf{E}+\boldsymbol{\varepsilon}_{\text {aux }}$, where $\boldsymbol{\varepsilon}_{\text {aux }}$ is the solution to the following problem of elastic equilibrium

$$
\begin{aligned}
& \mathbf{x} \in \tilde{\Omega} \backslash \Omega: \quad \nabla_{\mathbf{x}} \cdot\left(\mathbf{C}_{\text {aux }}: \varepsilon_{\text {aux }}\right)=\mathbf{0}, \\
& \mathbf{x} \in \tilde{\Omega} \backslash \Omega: \quad \boldsymbol{\varepsilon}_{\mathrm{aux}}(\mathbf{x})=\nabla_{\mathrm{x}}^{\mathrm{s}} \mathbf{u}_{\mathrm{aux}}, \\
& \mathbf{x} \in \partial \Omega: \quad \mathbf{u}_{\text {aux }}(\mathbf{x})=\mathbf{u}_{\mathrm{SUBC}}(\mathbf{x})-\mathbf{E} \cdot \mathbf{x}, \\
& \mathbf{x} \in \partial \tilde{\Omega}: \quad \mathbf{u}_{\text {aux }}(\mathbf{x})=\mathbf{0} .
\end{aligned}
$$

It should be emphasized that the above auxiliary problem [see Eqs. (D.1)] is merely introduced as a convenient way to construct a kinematically admissible strain which interpolates between displacements imposed at $\partial \Omega$ and $\partial \tilde{\Omega}$.

3. Outside $\tilde{\Omega}, \boldsymbol{\varepsilon}_{\text {trial }}=\mathbf{E}$.

It can readily be verified that $\boldsymbol{\varepsilon}_{\text {trial }} \in \mathbf{E}+\mathbb{K}^{\dagger}$. Using Eq. (9) to compute $\mathcal{W}\left(\boldsymbol{\varepsilon}_{\text {trial }}\right)$, and observing that (i) the integrand vanishes outside $\tilde{\Omega}$, and (ii) $\mathbf{C}=\mathbf{C}_{0}$ in $\tilde{\Omega} \backslash \Omega$, we find

$$
\mathcal{W}\left(\boldsymbol{\varepsilon}_{\text {trial }}\right)=\int_{\Omega}\left(\frac{1}{2} \varepsilon_{\mathrm{SUBC}}: \mathbf{C}: \boldsymbol{\varepsilon}_{\mathrm{SUBC}}-\mathbf{E}: \mathbf{C}_{0}: \boldsymbol{\varepsilon}_{\mathrm{SUBC}}+\frac{1}{2} \mathbf{E}: \mathbf{C}_{0}: \mathbf{E}\right)+\int_{\tilde{\Omega} \mid \Omega} \frac{1}{2} \boldsymbol{\varepsilon}_{\mathrm{aux}}: \mathbf{C}_{0}: \boldsymbol{\varepsilon}_{\mathrm{aux}} .
$$

From Hill's lemma in the bounded domain $\Omega$

$$
\int_{\Omega} \varepsilon_{\mathrm{SUBC}}: \mathbf{C}: \varepsilon_{\mathrm{SUBC}}=|\Omega| \overline{\boldsymbol{\sigma}_{\mathrm{SUBC}}: \varepsilon_{\mathrm{SUBC}}}=|\Omega| \overline{\boldsymbol{\sigma}_{\mathrm{SUBC}}}: \overline{\varepsilon_{\mathrm{SUBC}}}=|\Omega| \mathbf{E}: \mathbf{C}_{\mathrm{SUBC}}^{\mathrm{app}}: \mathbf{E},
$$

and

$$
\int_{\Omega} \mathbf{E}: \mathbf{C}_{0}: \varepsilon_{\mathrm{SUBC}}=|\Omega| \mathbf{E}: \mathbf{C}_{0}: \overline{\varepsilon_{\mathrm{SUBC}}}=|\Omega| \mathbf{E}: \mathbf{C}_{0}: \mathbf{E},
$$


which leads to the following expression of the potential energy $\mathcal{W}\left(\varepsilon_{\text {trial }}\right)$

$$
\mathcal{W}\left(\boldsymbol{\varepsilon}_{\text {trial }}\right)=\frac{1}{2}|\Omega| \mathbf{E}:\left(\mathbf{C}_{\mathrm{SUBC}}^{\mathrm{app}}-\mathbf{C}_{0}\right): \mathbf{E}+\int_{\tilde{\Omega} \backslash \Omega} \frac{1}{2} \boldsymbol{\varepsilon}_{\mathrm{aux}}: \mathbf{C}_{0}: \boldsymbol{\varepsilon}_{\text {aux }} .
$$
(34)]

Since $\boldsymbol{\varepsilon}_{\text {trial }} \in \mathbf{E}+\mathbb{K}^{\dagger}$, the principle of minimum potential energy (see Theorem 8) applies, and we have [using Eq.

$$
\frac{1}{2}|\Omega| \mathbf{E}:\left(\mathbf{C}_{\mathrm{MBC}}^{\mathrm{app}}\left(\mathbf{C}_{0}\right)-\mathbf{C}_{0}\right): \mathbf{E} \leq \mathcal{W}\left(\boldsymbol{\varepsilon}_{\text {trial }}\right),
$$

and finally

$$
0 \leq \mathbf{E}:\left(\mathbf{C}_{\mathrm{MBC}}^{\mathrm{app}}\left(\mathbf{C}_{0}\right)-\mathbf{C}_{\mathrm{SUBC}}^{\mathrm{app}}\right): \mathbf{E} \leq \frac{1}{|\Omega|} \int_{\tilde{\Omega} \mid \Omega} \boldsymbol{\varepsilon}_{\mathrm{aux}}: \mathbf{C}_{0}: \boldsymbol{\varepsilon}_{\mathrm{aux}} \leq \gamma_{\mathrm{SUBC}} \beta_{0},
$$

where

$$
\gamma_{\mathrm{SUBC}}=\frac{1}{|\Omega|} \int_{\tilde{\Omega} \mid \Omega} \boldsymbol{\varepsilon}_{\mathrm{aux}}: \boldsymbol{\varepsilon}_{\mathrm{aux}},
$$

It is recalled that $\boldsymbol{\varepsilon}_{\mathrm{aux}}$ solves a well-posed problem of linear elasticity in a bounded domain [see Eqs. (D.1)]; furthermore, this problem does not depend on $\mathbf{C}_{0}$. As a consequence, $\gamma_{\mathrm{SUBC}}$ is finite, and does not depend on $\mathbf{C}_{0}$.

Proof of Theorem 11. In order to invoke the principle of minimum complementary energy (see Theorem 9), we define on $\mathbb{R}^{d}$ the statically admissible trial stress $\sigma_{\text {trial }}$ as follows:

1. In $\Omega, \sigma_{\text {trial }}=\sigma_{\mathrm{KUBC}}$, where $\sigma_{\mathrm{KUBC}}$ is the stress arising from Problem KUBC [see Eqs. (1) and (3)]; by construction, $\overline{\sigma_{\mathrm{KUBC}}}=\mathbf{C}_{\mathrm{KUBC}}^{\mathrm{app}}: \mathbf{E}$.

2. In $\tilde{\Omega} \backslash \Omega, \sigma_{\text {trial }}=\mathbf{C}_{0}: \mathbf{E}+\sigma_{\text {aux }}$, where $\sigma_{\text {aux }}$ is the solution to the following problem of elastic equilibrium

$$
\begin{aligned}
\mathbf{x} \in \tilde{\Omega} \backslash \Omega: & \nabla_{\mathbf{x}} \cdot \sigma_{\text {aux }}=\mathbf{0}, \\
\mathbf{x} \in \tilde{\Omega} \backslash \Omega: & \sigma_{\text {aux }}(\mathbf{x})=\mathbf{C}_{\text {aux }}: \nabla_{\mathbf{x}}^{\mathrm{s}} \mathbf{u}_{\text {aux }}, \\
\mathbf{x} \in \partial \Omega: & \sigma_{\text {aux }}(\mathbf{x}) \cdot \mathbf{n}(\mathbf{x})=\left(\sigma_{\text {KUBC }}(\mathbf{x})-\overline{\boldsymbol{\sigma}}_{\text {KUBC }}\right) \cdot \mathbf{n}(\mathbf{x}), \\
\mathbf{x} \in \partial \tilde{\Omega}: & \sigma_{\text {aux }}(\mathbf{x}) \cdot \tilde{\mathbf{n}}(\mathbf{x})=\mathbf{0},
\end{aligned}
$$

where $\mathbf{n}$ (resp. $\tilde{\mathbf{n}}$ ) denotes the outward normal to $\partial \Omega$ (resp. $\partial \tilde{\Omega})$. In order to be well-posed, the above problem is complemented with appropriate additional displacement boundary conditions (not specified here).

3. Outside $\tilde{\Omega}, \sigma_{\text {trial }}=\mathbf{C}_{0}: \mathbf{E}$.

It can readily be verified that $\sigma_{\text {trial }} \in \mathbf{C}_{0}: \mathbf{E}+\mathbb{S}^{\dagger}$. Using Eq. (10) to compute $\mathcal{W}^{*}\left(\sigma_{\text {trial }}\right)$, and observing that (i) the integrand vanishes outside $\tilde{\Omega}$ and (ii) $\mathbf{S}=\mathbf{S}_{0}$ in $\tilde{\Omega} \backslash \Omega$, we find

$$
W^{*}\left(\sigma_{\text {trial }}\right)=\int_{\Omega}\left(\frac{1}{2} \sigma_{\mathrm{KUBC}}: \mathbf{S}: \boldsymbol{\sigma}_{\mathrm{KUBC}}-\mathbf{E}: \sigma_{\mathrm{KUBC}}+\frac{1}{2} \mathbf{E}: \mathbf{C}_{0}: \mathbf{E}\right)+\int_{\tilde{\Omega} \mid \Omega} \frac{1}{2} \sigma_{\text {aux }}: \mathbf{S}_{0}: \sigma_{\text {aux }} .
$$

From Hill's lemma in the bounded domain $\Omega$

$$
\int_{\Omega} \sigma_{\mathrm{KUBC}}: \mathbf{S}: \sigma_{\mathrm{KUBC}}=|\Omega| \overline{\boldsymbol{\sigma}_{\mathrm{KUBC}}: \boldsymbol{\varepsilon}_{\mathrm{KUBC}}}=|\Omega| \overline{\sigma_{\mathrm{KUBC}}}: \overline{\varepsilon_{\mathrm{KUBC}}}=|\Omega| \mathbf{E}: \mathbf{C}_{\mathrm{KUBC}}^{\mathrm{app}}: \mathbf{E},
$$

and

$$
\int_{\Omega} \mathbf{E}: \sigma_{\mathrm{KUBC}}=|\Omega| \mathbf{E}: \overline{\boldsymbol{\sigma}}_{\mathrm{KUBC}}=|\Omega| \mathbf{E}: \mathbf{C}_{\mathrm{KUBC}}^{\mathrm{app}}: \mathbf{E}
$$

which leads to the following expression of the complementary energy $\mathcal{W}^{*}\left(\sigma_{\text {trial }}\right)$

$$
\mathcal{W}^{*}\left(\sigma_{\text {trial }}\right)=\frac{1}{2}|\Omega| \mathbf{E}:\left(\mathbf{C}_{0}-\mathbf{C}_{\mathrm{KUBC}}^{\mathrm{app}}\right): \mathbf{E}+\int_{\tilde{\Omega} \mid \Omega} \frac{1}{2} \sigma_{\text {aux }}: \mathbf{S}_{0}: \sigma_{\text {aux }} .
$$


Since $\sigma_{\text {trial }} \in \mathbf{C}_{0}: \mathbf{E}+\mathbb{S}^{\dagger}$, the principle of minimum complementary energy (see Theorem 9) applies, and we have [using Eq. (38)]

$$
\frac{1}{2}|\Omega| \mathbf{E}:\left(\mathbf{C}_{0}-\mathbf{C}_{\mathrm{MBC}}^{\mathrm{app}}\left(\mathbf{C}_{0}\right)\right): \mathbf{E} \leq \mathcal{W}^{*}\left(\sigma_{\text {trial }}\right)
$$

and finally

$$
0 \leq \mathbf{E}:\left(\mathbf{C}_{\mathrm{KUBC}}^{\mathrm{app}}-\mathbf{C}_{\mathrm{MBC}}^{\mathrm{app}}\left(\mathbf{C}_{0}\right)\right): \mathbf{E} \leq \frac{1}{|\Omega|} \int_{\tilde{\Omega} \backslash \Omega} \sigma_{\mathrm{aux}}: \mathbf{S}_{0}: \boldsymbol{\sigma}_{\mathrm{aux}} \leq \frac{\gamma_{\mathrm{KUBC}}}{\alpha_{0}},
$$

where

$$
\gamma_{\mathrm{KUBC}}=\frac{1}{|\Omega|} \int_{\tilde{\Omega} \backslash \Omega} \sigma_{\mathrm{aux}}: \sigma_{\mathrm{aux}} .
$$

It is recalled that $\sigma_{\text {aux }}$ solves a well-posed problem of linear elasticity in a bounded domain [see Eqs. (D.2)]; furthermore, this problem does not depend on $\mathbf{C}_{0}$. As a consequence, $\gamma_{\mathrm{KUBC}}$ is finite, and does not depend on $\mathbf{C}_{0}$.

\section{Appendix E. On the principle of Hashin and Shtrikman}

In the present section, two alternative expressions of the bilinear form $a$ introduced in Eq. (43) are proposed [see Eqs (E.2) and (E.4)]. Eq. (44), upon which the proof of Theorem 12 is based, is a direct consequence of these two alternative expressions.

Lemma 2. Let $\varpi_{1}, \varpi_{2} \in \mathbb{L}^{2}(\Omega), \boldsymbol{\varepsilon}_{i}=-\boldsymbol{\Gamma}_{0}^{\infty} *\left(\varpi_{i}-\chi \bar{\varpi}_{i}\right)$ and $\boldsymbol{\sigma}_{i}=\mathbf{C}_{0}: \boldsymbol{\varepsilon}_{i}+\varpi_{i}(i=1,2)$. Then

$$
\overline{\varpi_{1}:\left(\boldsymbol{\Gamma}_{0}^{\infty} *\left(\varpi_{2}-\chi \bar{\varpi}_{2}\right)\right)}=|\Omega|^{-1}\left\langle\boldsymbol{\varepsilon}_{1}, \mathbf{C}_{0}: \boldsymbol{\varepsilon}_{2}\right\rangle,
$$

and

$$
a\left(\varpi_{1}, \varpi_{2}\right)=\overline{\varpi_{1}:\left(\mathbf{C}-\mathbf{C}_{0}\right)^{-1}: \varpi_{2}}+|\Omega|^{-1}\left\langle\boldsymbol{\varepsilon}_{1}, \mathbf{C}_{0}: \boldsymbol{\varepsilon}_{2}\right\rangle
$$

Proof of Lemma 2. Recalling that $\varpi_{1}$ is supported in $\Omega$

$$
\begin{aligned}
|\Omega| \overline{\varpi_{1}:\left(\Gamma_{0}^{\infty} *\left(\varpi_{2}-\chi \overline{\boldsymbol{\varpi}}_{2}\right)\right)} & =\left\langle\boldsymbol{\varpi}_{1}, \boldsymbol{\Gamma}_{0}^{\infty} *\left(\varpi_{2}-\chi \overline{\boldsymbol{\varpi}}_{2}\right)\right\rangle=-\left\langle\boldsymbol{\varpi}_{1}, \boldsymbol{\varepsilon}_{2}\right\rangle \\
& =\left\langle\mathbf{C}_{0}: \boldsymbol{\varepsilon}_{1}-\boldsymbol{\sigma}_{1}, \boldsymbol{\varepsilon}_{2}\right\rangle=\left\langle\boldsymbol{\varepsilon}_{1}, \mathbf{C}_{0}: \boldsymbol{\varepsilon}_{2}\right\rangle-\left\langle\boldsymbol{\sigma}_{1}, \boldsymbol{\varepsilon}_{2}\right\rangle
\end{aligned}
$$

and the last term vanishes from Theorem 5 (since $\sigma_{i} \in \mathbb{S}^{\dagger}$ and $\boldsymbol{\varepsilon}_{i} \in \mathbb{K}^{\dagger}, i=1,2$, see Sec. 4.2), which proves Eq. (E.1).

Lemma 3. Let $\varpi_{1}, \varpi_{2} \in \mathbb{L}^{2}(\Omega)$, $\boldsymbol{\varepsilon}_{i}=-\boldsymbol{\Gamma}_{0}^{\infty} *\left(\varpi_{i}-\chi \bar{\varpi}_{i}\right)$ and $\boldsymbol{\sigma}_{i}=\mathbf{C}_{0}: \boldsymbol{\varepsilon}_{i}+\varpi_{i}(i=1,2)$. Then

$$
\overline{\varpi_{1}:\left(\boldsymbol{\Gamma}_{0}^{\infty} *\left(\varpi_{2}-\chi \bar{\varpi}_{2}\right)\right)}=-|\Omega|^{-1}\left\langle\sigma_{1}, \mathbf{S}_{0}: \sigma_{2}\right\rangle+\overline{\varpi_{1}: \mathbf{S}_{0}: \varpi_{2}},
$$

and

$$
a\left(\varpi_{1}, \varpi_{2}\right)=\overline{\varpi_{1}: \mathbf{S}_{0}:\left(\mathbf{S}_{0}-\mathbf{S}\right)^{-1}: \mathbf{S}_{0}: \varpi_{2}}-|\Omega|^{-1}\left\langle\boldsymbol{\sigma}_{1}, \mathbf{S}_{0}: \sigma_{2}\right\rangle
$$

Proof of Lemma 3. First, recalling that $\varpi_{1}$ is supported in $\Omega$

$$
\begin{aligned}
|\Omega| \overline{\varpi_{1}:\left(\boldsymbol{\Gamma}_{0}^{\infty} *\left(\varpi_{2}-\chi \overline{\boldsymbol{\varpi}}_{2}\right)\right)} & =\left\langle\varpi_{1}, \boldsymbol{\Gamma}_{0}^{\infty} *\left(\varpi_{2}-\chi \bar{\varpi}_{2}\right)\right\rangle=\left\langle\varpi_{1}, \mathbf{S}_{0}:\left(\varpi_{2}-\boldsymbol{\sigma}_{2}\right)\right\rangle \\
& =-\left\langle\varpi_{1}, \mathbf{S}_{0}: \boldsymbol{\sigma}_{2}\right\rangle+|\Omega| \overline{\varpi_{1}: \mathbf{S}_{0}: \varpi_{2}}
\end{aligned}
$$


then

$$
\left\langle\varpi_{1}, \mathbf{S}_{0}: \sigma_{2}\right\rangle=\left\langle\sigma_{1}-\mathbf{C}_{0}: \varepsilon_{1}, \mathbf{S}_{0}: \sigma_{2}\right\rangle=\left\langle\sigma_{1}, \mathbf{S}_{0}: \sigma_{2}\right\rangle-\left\langle\sigma_{2}, \varepsilon_{1}\right\rangle
$$

where the last term vanishes from Theorem 5 (since $\sigma_{i} \in \mathbb{S}^{\dagger}$ and $\boldsymbol{\varepsilon}_{i} \in \mathbb{K}^{\dagger}, i=1,2$, see Sec. 4.2); thus, Eq. (E.3) is proved. Proof of Eq. (E.4) follows from the identity (Willis, 1977)

$$
\left(\mathbf{C}-\mathbf{C}_{0}\right)^{-1}: \mathbf{C}_{0}=\mathbf{S}_{0}:\left(\mathbf{S}_{0}-\mathbf{S}\right)^{-1}-\mathbf{I}
$$

resulting in

$\overline{\varpi_{1}:\left(\mathbf{C}-\mathbf{C}_{0}\right)^{-1}: \varpi_{2}}=\overline{\varpi_{1}: \mathbf{S}_{0}:\left(\mathbf{S}_{0}-\mathbf{S}\right)^{-1}: \mathbf{S}_{0}: \varpi_{2}}-\overline{\varpi_{1}: \mathbf{S}_{0}: \varpi_{2}}$ 